\title{
MAPPING OF THE DESCRIPTIVE LOGIC INTO RDF USING BINARY RELATIONAL DATA MODEL
}

\begin{abstract}
This paper is dedicated to the data integration problem. The descriptive logic and the relational data model are at the heart of a study. They have been used to create a mapping method on the theoretical level. The previous studies are continued in this paper to prove on practice a mapping creation method between the descriptive logic and the binary relational data model, which is a part of a mapping method. The method uses the binary relational data model as an integrating model. The task to prove the theoretical mapping method on practice was formulated. A question how to map the binary relational data model into RDF-triples was considered. A brief overview of the R2R ML conversion tool was given. Triple maps were created to convert a conceptual information model of descriptive logic into RDF triplets with the help of R2R ML. Also, triples maps are described to convert basic mapping mechanisms into RDF with the help of R2R ML.

Key words: binary relational data model, description logic, mapping, RDF, DL, RM ${ }^{2}$, ALC, OWL.
\end{abstract}

\section{Introduction}

A complex problem of data integration in the semantic web exists in the modern scientific field of research. An analysis of this problem can be found in the [1]. A task to establish an interaction between a descriptive logic (DL) and a relational data model (RDM) arises as a part of solution of the data integration problem. Such interaction is called mapping. To establish an interaction means to create mapping mechanisms between the DL and the RDM. A series of studies [1-7] is dedicated to the analysis and solution of the mapping creation problem. A binary relational data model $\left(\mathbf{R M}^{\mathbf{2}}\right)$ [6] was created as a result of this series. The main task of $\mathrm{RM}^{2}$ is to be an integrating model for creating mappings. How to map the descriptive logic ALC and its main components into $\mathrm{RM}^{2}$ was described in the study [8] as well as how to map the classical RDM into $\mathrm{RM}^{2}$. This approach was described purely on a theoretical level. Until now, the lack of any practical approbation was a significant drawback of the proposed results.

A method to test mappings between DL and $\mathrm{RM}^{2}$ with the help of RDF graphs is proposed in this paper. The main idea is to map DL-to-RDM conversion formulas into $\mathrm{RDF}$, and then to test them for workability within the unified RDF framework. The results of mapping DL expressions into RDF using OWL 2 were published in [9]. This study focuses on creation of mappings for RDM expressions into RDF using R2R ML.
Section 1 is dedicated to the problem statement. Section 2 provides a short overview of the R2R ML. Section 3 summarizes the main theoretical aspects of the DL-RDM mapping method. Section 4 describes the $\mathrm{RM}^{2}$ to RDF mapping rules using R2R ML. Section 5 contains conclusions.

\section{Problem statement}

A theoretical approach how to describe mappings between DL and RDM is presented in a series of studies [1-9]. A binary relational data model $\left(\mathrm{RM}^{2}\right)$ is proposed as an integrating model. The approbation task of this approach is based on a number of facts.

Firstly, it is known that DL is the mathematical basis of any ontology description language. Thus, all the constructors of concepts and roles of the underlying DL are reflected in the toolbox of the corresponding language. OWL 2 is not an exception.

Secondly, only binary connections are allowed in $\mathrm{RM}^{2}$. Both binary and n-ary connections are allowed in a classical RDM. A result of [10] seems to indicate that any n-ary relation can be represented by a set of binary ones. Thus, any classic RDM can be expressed with $\mathrm{RM}^{2}$. A way how to convert $\mathrm{RDM}$ into $\mathrm{RM}^{2}$ is described in [6].

The idea of testing is not new. The statements of the theory being proved are transformed into statements of the established theory. The converted expressions are then checked for truth within the well-established

(C) I.S. Chystiakova, 2021 
theoery using its own methods and properties. If the final expression is true in the existing theory, then the original expression is also true in the study area.

Thus, the problem statement ti test mappings between DL and RDM is formulated as follows. On the one hand, DL statements (expressed in OWL 2) are mapped to the RDF triplets using OWL 2-to-RDF conversion rules. On the other hand, relational database (RDB) expressions are mapped to the RDF triplets using R2R ML. The resulting RDF triplets constitute a set of RDF graphs. The resulting graphs are compared for equivalence.

The implementation idea is schematically shown in Figure 1.

Figure1. Approbation scheme of the mapping

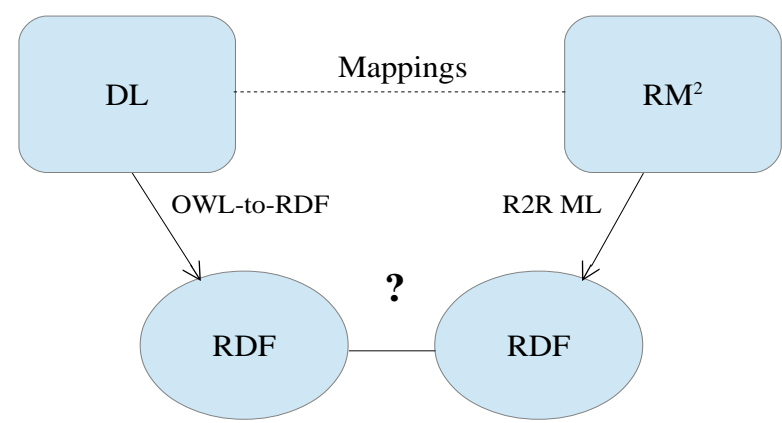

method between DL and $\mathrm{RM}^{2}$

OWL 2-to-RDF conversion rules have official W3C status [11]. R2R ML also has official W3C status [12].

The algorithm for testing mappings between DL and $\mathrm{RM}^{2}$ is as follows:

Step 1. There is a DL expression. It is mapped into the RM2 statement.

Step 2. The statement is mapped intto an OWL 2-expression from the DL side.

Step 3. OWL 2-expression (step 2) is converted to RDF-triples that form an RDFgraph. OWL 2-to-RDF rules are used for mapping.

Step 4. The statement is formulated in terms of RDB from the RM2 side.

Step 5. RDB expression is converted to the RDF-triples that form an RDF-graph. $\mathrm{R} 2 \mathrm{R}$ ML is used to create mappings.

Step 6. The RDF-graph (step 3) is compared for equivalence with another RDF- graph (step 5). If they are equivalent, then the DL-to-RM2 mapping formula is true.

The mappings from step 1 are described in [8] on the theoretical level. Also, they are briefly described in section 3 . The mappings from steps 2 and 3 are described in article [9]. The current work will present a way to perform transformations from steps 4 and 5. The comparison from step 6 remains in the field of future research.

It is known that OWL 2 is based on the SROIQ descriptive logic. Thus, the OWL 2-to-RDF mapping area is limited to only those operations that are present in DL SROIQ. DL SROIQ syntax include the following: DL ALC syntax, DL axiomatics, numerical constraints, nominals, and inverse roles. The theoretical part of DL-to-RM ${ }^{2}$ mappings has been worked out for several role constructors. The issue of mapping some of the role constructors in $\mathrm{RDF}$ (except the inverse role) remains open.

There are a number of features in R2R ML. It allows you to transform the structure and integrity constraints of an RDB into RDF triplets. However, there are some features of mapping the manipulative part of RDM into RDF. Any operation can be mapped only as part of an SQL query. Each SQL query is represented as a logical table within a triples map generated for such table. Thus, there are no mechanisms to transform directly each individual operation of relational algebra (RA) within R2R ML itself. A small overview of R2RML is presented in section 2.

A key question of the approbation task is to prove the equivalence of graphs, obtained as a result of pairwise mapping of the $\mathrm{DL}$ and $\mathrm{RM}^{2}$ statements. The results of [13] demonstrate that RDF-graph is a special case of a regular graph. This means that the question of equivalence is reduced to proving their isomorphism. In the work [13] RDF-graph is analyzed as a special case of a usual graph. Several criteria for graph isomorphism in the general case are also studied. Based on these criteria, three necessary and sufficient conditions for the RDF-graphs equivalence of are formulated. They are as follows: 
1. Equal number of vertices. Both graphs must contain the same number of vertices, otherwise they are not isomorphic.

2. Vertices equivalence. Each vertex of one graph must have an equivalent in the other graph in a pairwise comparison. Otherwise, such graphs are not isomorphic.

3. Ribs equivalence. Each edge of one graph must have an equivalent in the other graph in a pairwise comparison. Otherwise, such graphs are not isomorphic.

Reducing a graph to a self-isomorphic remains the last question in the problem framework. As a result of mappings, the following situation may arise at the RDF level. The vertex of one graph will semantically correspond to a subgraph of the comparable graph. Such a subgraph can consist of several vertices connected by edges. This situation is possible, since a large number of anonymous (empty) nodes appears during the mapping process. Such nodes have their own semantic purpose. Thus, the question of reducing an RDF-graph to the self-isomorphic remains open.

\section{Preliminaries: R2R ML Overview}

Here is a brief overview of the R2R ML. It will be used in Section 4 to map $\mathrm{RM}^{2}$ expressions into RDF.

The R2R ML has a development history. Tim Berners-Lee published an article [14] in 1998. It was entitled as "Relational Databases on the Semantic Web". This paper discusses the concept of presenting any database in the semantic web. Its main idea is to use RDF as an ER model. The ER model establishes a correlation between relational database elements and RDF-triples. The author of the concept proposes to use an XML-format of serialization of RDF-triples. The paper also presents the "bottlenecks" of mapping of the RDB information into RDF.

Then, in 2007, the conference "W3C Workshop on RDF Access to Relational Databases" was held [15]. It was dedicated to the presentation of ordinary relational data in $\mathrm{RDF}$ format, as well as the use of RDF in RDB queries. The W3C RDB2RDF Incubator Group was established in 2009 and operated until 2012. It declared the following goals:
- to study and classify existing approaches of mapping the relational data in $\mathrm{RDF}$;

- to determine the need for standardization of this area;

- to determine the mechanisms of generation the RDF-triples from one or more relational databases without the loss of information;

- to study the possibility of mapping OWL classes into relational data, taking into account the developed approach.

As a result, a document with official R2R ML recommendations was received and published. Figure 2 shows the chronology of R2R ML development [16].

Now it is necessary to consider the main standings of R2R ML. It's important to understand how RDF-triples are formed from relational data. Let's turn to the official documentation [12].

R2R ML - is a language for expressing customized mappings from relational databases to RDF datasets. It defines the mapping of the relational database into the RDF. An R2RML mapping refers to logical tables to retrieve data from the input database. The input to an $\mathrm{R} 2 \mathrm{R} \mathrm{ML}$ mapping is called the input database. Figure 3 highlights a UML diagram of the R2R ML language overview. The picture is taken from the official $\mathrm{R} 2 \mathrm{R} M L$ website.

An R2R ML mapping - is a structure that consists of one or more triples maps. A triples map is a rule that maps each row in the logical table to a number of RDF triples. The rule has two main parts: a subject map and multiple predicate-object map.

A subject map generates the subject of all RDF triples that will be generated from a logical table row. The subjects are often IRIs that are generated from the primary key column(s) of the table.

Predicate-object maps in turn consist of predicate maps and object maps. Sometimes predicate-object map additionally has referencing object maps. 

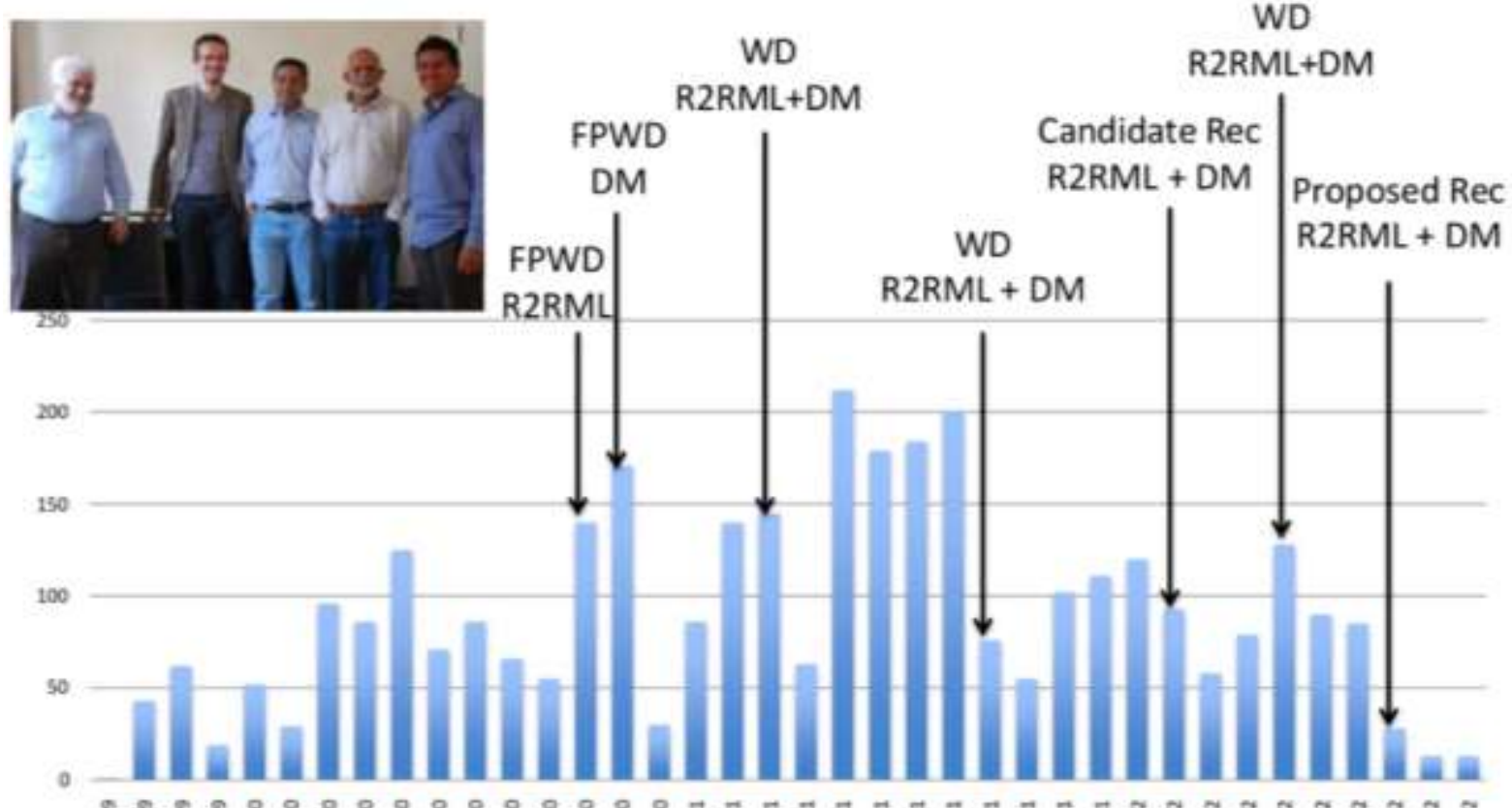

WD

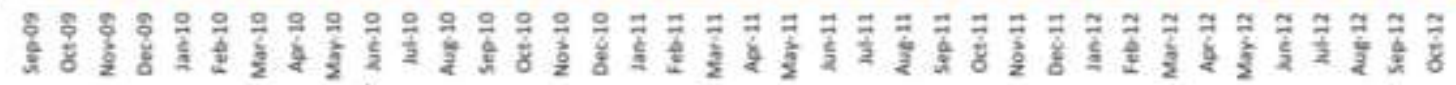

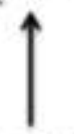

First F2F

@Semtech 2010

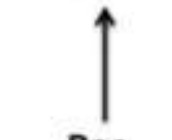

$\operatorname{Rec}$

$R 2 R M L+D M$

Photo from cygri http://www.flickr.com/photos/cygri/4719458268/

Figure 2. Chronology of the R2R ML development

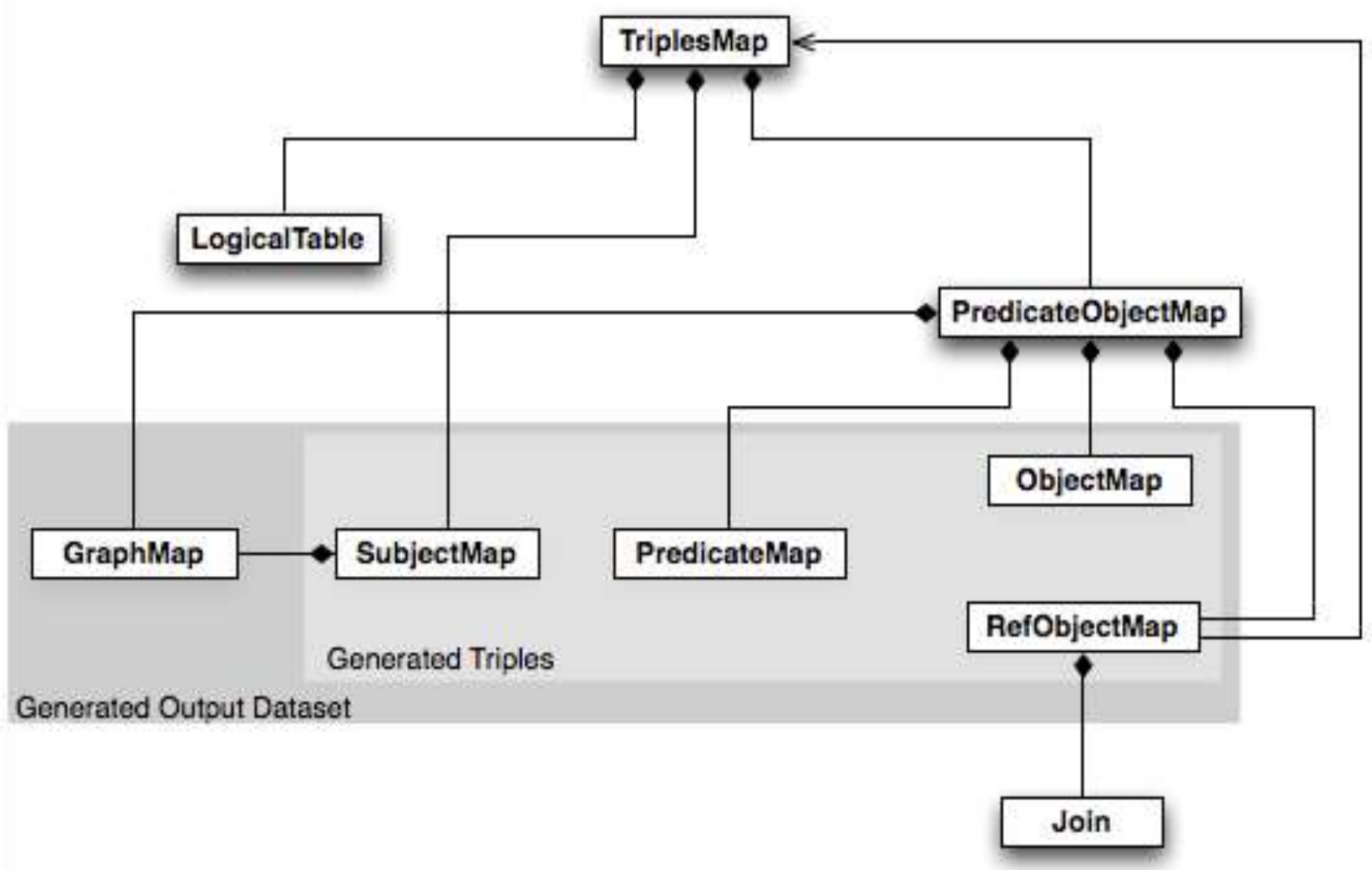

Figure 3. R2R ML overview 
Triples are produced as follows. Subject map is combined with a predicate map and object map. These three are applied to each logical table row. By default, all RDF triples are in the default graph of the output dataset. A triples map can contain graph maps. Such graph maps place some or all of the triples into named graphs instead of the default graph.

Triples map (fig. 2) consists of the three main components: a logical table, a subject map, a predicate-object map. In detail each of them is as follows.

A logical table is a tabular SQL query result that is to be mapped to RDF triples. In simple words a logical table is what will be displayed. It can take one of the following three forms:

- $\quad$ SQL base table;

- SQL view;

- $\quad$ R2R ML view (a valid SQL query). It got its name because it only emulates a SQL view without modifying the database.

A logical table row is a row in a logical table. It can be a row of a base SQL table or SQL view. It can also be a row of an R2R ML view obtained with an SQL query.

A column name is the name of a column of a logical table. A column name must be a valid $S Q L$ identifier. For example, the name of a SQL object, such as a column, table, view, schema, or catalog. Column names do not include any qualifying table, view or schema names.

The logical table in the triples map is written using one of the properties:

- rr:tableName - specifies the table or view name of the base table or view. Its value must be a valid schema-qualified name. It is a sequence of one, two or three valid SQL identifiers, separated by the dot character (“.”). The three identifiers name, respectively, a catalog, a schema, and a table or view. If no catalog or schema is specified, then the default catalog and default schema of the SQL connection are assumed.

- rr:sqlQuery and rr:sqlVersion defines R2R ML view and SQL query version. R2R ML view is a logical table whose contents are the result of executing a SQL query against the input database. If rr:sqlVersion property is absent, then the rr:sqlQuery property value conforms to Core SQL 2008.

Before considering the subject maps and the predicate-object maps, it's necessary to give a number of definition.

An RDF term is either an IRI, or a blank node, or a literal.

A term map is a function that generates an RDF term from a logical table row. The result of that function is a generated RDF term. Term maps are used to generate the subjects, predicates and objects of the RDF triples. In turn, RDF triples are generated by a triples map. Consequently, there are several kinds of term maps, depending on where in the mapping they occur: subject maps, predicate maps, object maps and graph maps. The referenced columns of a term map are the set of column names referenced in the term map. They depend on the type of term map.

A subject map is a term map. It specifies a rule for generating the subjects of the RDF triples.

In the triples map, the subject map is specified as follows:

- rr:subjectMap is a property, which value must be a specific subject map;

- rr:subject is a shortcut constant property whose value is IRI.

The subject map can contain the rr:class property. Its value must be an IRI, which is called class IRI. In this case, the RDF expression generated by the subject map will look like this. For each subject, a triple is created with the rdf:type predicate and the rr:class property as object value. A subject map can contain several class IRIs at the same time. There are cases when the class IRI must be computed based on the contents of source database. In such situations, a predicate object map is used. The predicate value is indicated by rdf: type. The value of an object is set through a non-constant object map.

A predicate-object map is a function that creates one or more predicate-object pairs for each logical table row of a logical table. It is used in conjunction with a subject map to generate RDF triples in a triples map. A predicate-object map is represented by a resource that references: one or 
more predicate maps and one or more object maps.

A predicate map is a term map. It can be defined in two ways:

- rr:predicateMap is a property, whose value must be a predicate map;

- rr:predicate is a constant shortcut property whose value is IRI.

An object map is a term map. It can be defined in two ways:

- $\quad r r$ :objectMap is a property, whose value must be either an object map, or a referencing object map;

- rr:object is a constant shortcut property whose value is IRI or literal.

A referencing object map allows using the subjects of another triples map as the objects generated by a predicate-object map. Since both triples maps may be based on different logical tables, this may require a join between the logical tables. However, the join condition (one or more joins) is optional.

A referencing object map is represented by the following resources:

- rr:parentTriplesMap is a property, whose value must be a triples map. Such triples map is known as the referencing object map's parent triples map. The value of object will be extracted exactly from the parent triples map.

- rr:joinCondition is a property whose values must be join conditions options.

A join condition is represented by a resource that has exactly one value for each of the following two properties:

- $\quad r r$ :child is a property, whose value is known as the join condition's child column. It must be a column name that exists in the logical table of the triples map (that contains the referencing object map).

- $\quad$ rr:parent is a property whose value is known as the join condition's parent column. It must be a column name that exists in the logical table of parent triples map (of the referencing object map's). The name of the parent triples map was specified in the rr:parentTriplesMap property.
Here is one of the examples given in the description of the R2R ML standard [12]. The following example database consists of two tables, EMP (table 1) and DEPT (table 2), with one row each:

Table 1

EMP

\begin{tabular}{|l|l|l|l|}
\hline $\begin{array}{l}\text { EMPNO } \\
\text { Integer } \\
\text { primary } \\
\text { key }\end{array}$ & $\begin{array}{l}\text { EName } \\
\text { Varchar } \\
(100)\end{array}$ & $\begin{array}{l}\text { Job } \\
\text { Varchar } \\
(20)\end{array}$ & $\begin{array}{l}\text { DEPTNO } \\
\text { Integer } \\
\text { references } \\
\text { DEPT } \\
\text { (DEPTNO) }\end{array}$ \\
\hline 7369 & Smith & Clerk & 10 \\
\hline
\end{tabular}

Table 2

DEPT

\begin{tabular}{|l|l|l|}
\hline $\begin{array}{l}\text { DEPTNO } \\
\text { Integer } \\
\text { primary key }\end{array}$ & $\begin{array}{l}\text { DName } \\
\text { Varchar (30) }\end{array}$ & $\begin{array}{l}\text { Loc } \\
\text { Varchar } \\
(100)\end{array}$ \\
\hline 10 & Appserver & New York \\
\hline
\end{tabular}

The following R2R ML mapping document will produce the desired triples from the EMP table:

@prefix $r r:$ <http://www.w3.org/ns/r2rml\#>.

@prefix ex: 〈http://example.com/ns\#>.

<\#ЕтрMap>

rr:logicalTable [ rr:tableName "EMP" ];

rr:subjectMap [

rr:template

"http://data.example.com/employee/\{EMPNO\}"; rr:class ex:Employee;

];

rr:predicateObjectMap [

rr:predicate ex:name;

rr:objectMap [ rr:column "EName" ];

];

rr:predicateObjectMap I

rr:predicate ex:job;

rr:objectMap [ rr:column "Job" ];

];

rr:predicateObjectMap [

rr:predicate ex:department;

rr:objectMap [

rr:parentTriplesMap 〈\#DeptMap〉;

rr:joinCondition [ 
rr:child "DEPTNO";

rr:parent "DEPTNO";

];

].

The definition of a triples map that generates the desired DEPT triples follows.

<\#DeptTableView> rr:sqlQuery "'"'"

SELECT DEPTNO, DName, Loc, (SELECT COUNT(*) FROM EMP WHERE EMP.DEPTNO=DEPT.DEPTNO) AS Staff

FROM DEPT;

"'"'".

<\#DeptMap>

rr:logicalTable 〈\#DeptTableView〉;

rr:subjectMap [

rr:template

"http://data.example.com/department/\{DEPTNO\} ";

rr:class ex:Department;

];

rr:predicateObjectMap [

rr:predicate ex:name;

rr:objectMap [ rr:column "DName"];

];

rr:predicateObjectMap [

rr:predicate ex:location;

rr:objectMap [ rr:column "Loc"];

];

rr:predicateObjectMap [

rr:predicate ex:staff;

rr:objectMap [ rr:column "Staff" ];

].

The desired RDF triples to be produced from this database are as follows:

<http://data.example.com/employee/7369

$>$ rdf:type ex:Employee.

$<$ http://data.example.com/employee/7369

$>$ ex:name "Smith".

$<$ http://data.example.com/employee/7369

$>$ ex:job "Clerk".

$<$ http://data.example.com/employee/7369

$>$ ex:department <http://data.example.com/depart ment/10>.

<http://data.example.com/department/10

$>$ rdf:type ex:Department.

$<$ http://data.example.com/department/10

> ex:name "Appserver".
$<$ http://data.example.com/department/10

$>$ ex:location "New York".

$<$ http://data.example.com/department/10

$>$ ex:staff 1 .

\section{Preliminaries: mapping DL into $\mathbf{R M}^{2}$}

The approbation task of testing mappings between DL and RDM is based on a series of theoretical studies [1-7]. Here is a brief summary of them. This summary will be used in Section 4 to create mappings from $\mathrm{RM}^{2}$ to RDF.

Binary Relational Data Model $\left(\mathrm{RM}^{2}\right)$ [6] was developed to address the issue of establishing relations between DL and RDM. It has several advantages over the classic RDM by Codd [17]. For example, $\mathrm{RM}^{2}$ contains support for the open world assumption, while classical RDM works according to epy closed world assumption. Unlike Codd's $\mathrm{RDM}, \mathrm{RM}^{2}$ supports the implementation of DL constructors and concepts and roles axioms. The $\mathrm{RM}^{2}$ is described in detail in [6].

To describe mappings, the first step is to build a conceptual information model of descriptive logic. The main task of the conceptual information model of any subject area is to define the basic concepts and to describe their properties and relations. The ER language is one of the most used for this purpose. It operates with the concepts of entity, attribute and relation. The Barker dialect [18] of the ER language was used to describe the conceptual information model of descriptive logic (Fig. 4).

There are three basic entities in the model:

- Concept - to present DL concepts

- Role - to present DL roles

viduals

- CIndividual - to present DL indi-

Each entity has a single attribute that is called "Name". This attribute uniquely identifies the entities. The rest of entities are relation entities. They represent binary relations between basic entities. Link entities do not have their own attributes and are uniquely identified only by their links. 


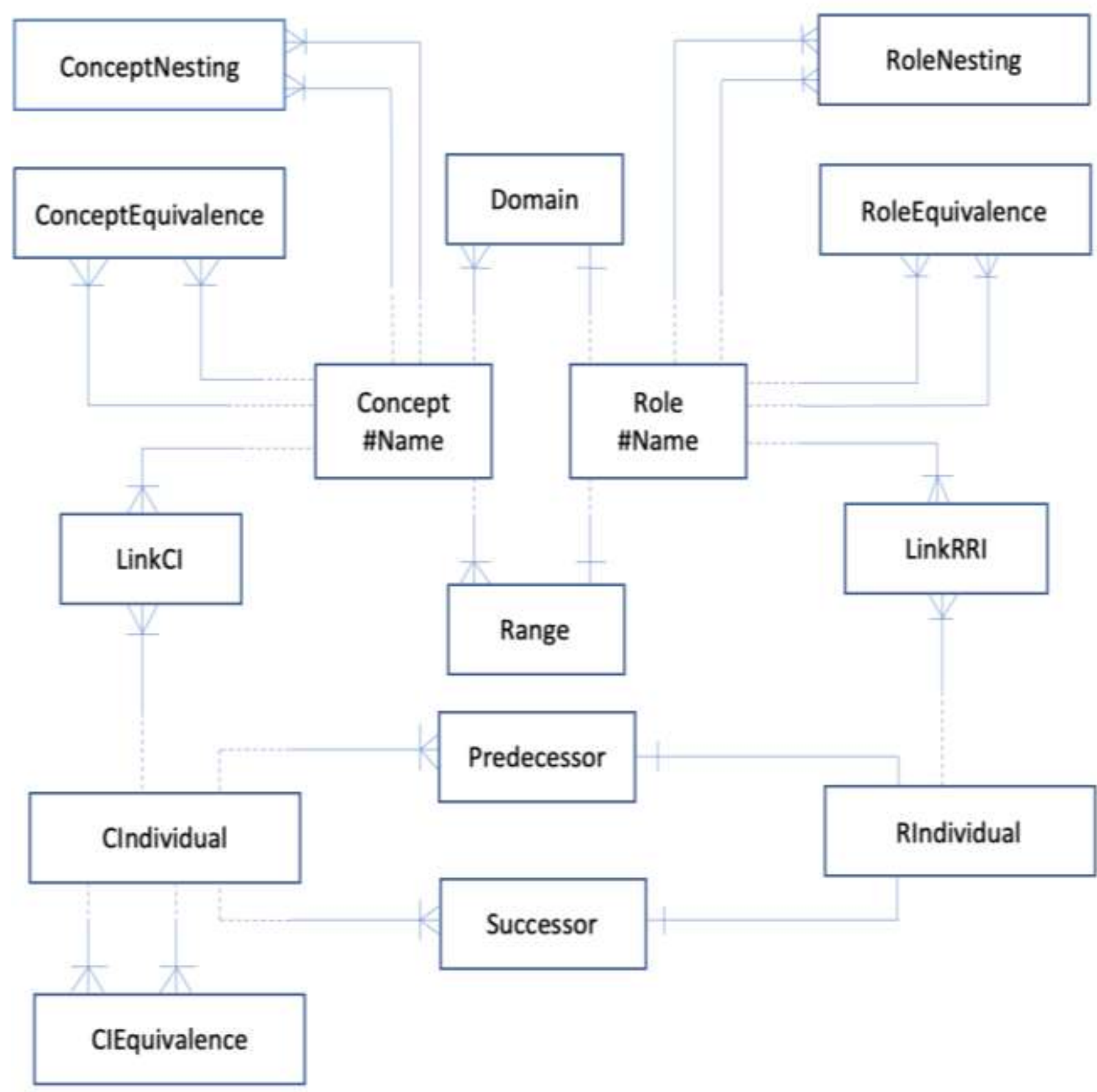

Figure 4. Conceptual information model of descriptive logic

The $\mathrm{RM}^{2}$ scheme was built according to the given ER-model. The transformation algorithm described in $[2,6,8]$ was used to construct the scheme. Additionally, the idea of placeholder attributes was used to represent the primary keys. This idea firstly was proposed by E. Codd [17], the founder of RDM. Each entity is represented as a $\mathrm{RM}^{2}$ relationship, since the constructed ERscheme fits the $3 \mathrm{NF}$ requirement. The name of the relationship is the same as the name of the entity. Table 3 highlights all the ER- model entities with their descriptions and the corresponding relationships with the list of attributes.

To describe mappings, the second step is to express DL ALC in $\mathrm{RM}^{2}$. ALC is the simplest variant of DL. It is included in all widely used dialects of other DLs. It should be recalled, that description logics uses constructs that have semantics given in predicate logic. The ALC semantics is defined through the concept of interpretation. 
ER-model entities with their descriptions and corresponding relationships

\begin{tabular}{|c|c|c|}
\hline $\begin{array}{l}\text { Entity (rela- } \\
\text { tionship) name }\end{array}$ & Entity description & Relationship attributes with description \\
\hline Concept & Presents concepts & $\begin{array}{l}\text { CPK - primary key } \\
\text { Name - concept name }\end{array}$ \\
\hline Role & Present roles & $\begin{array}{l}\text { RPK - primary key } \\
\text { Name - role name } \\
\text { IsTransitive - is role transitive }\end{array}$ \\
\hline CIndividual & Present individuals & $\begin{array}{l}\text { CIPK - primary key } \\
\text { Name - individual name }\end{array}$ \\
\hline RIndividual & Pesent role individuals & RIPK - primary key \\
\hline Domain & Present role domain & $\begin{array}{l}\text { CFK - foreign key on Concept } \\
\text { RFK - foreign key on Role }\end{array}$ \\
\hline Range & Present role range & $\begin{array}{l}\text { CFK - foreign key on Concept } \\
\text { RFK - foreign key on Role }\end{array}$ \\
\hline LinkCI & $\begin{array}{l}\text { Allows many-to-many rela- } \\
\text { tions between concepts and } \\
\text { individuals }\end{array}$ & $\begin{array}{l}\text { CFK - foreign key on Concept } \\
\text { CIFK - foreign key on CIndividual }\end{array}$ \\
\hline LinkRRI & $\begin{array}{l}\text { Allows many-to-many rela- } \\
\text { tions between roles and their } \\
\text { instances }\end{array}$ & $\begin{array}{l}\text { RFK - foreign key on Concept } \\
\text { RIFK - foreign key on RIndividual }\end{array}$ \\
\hline Predecessor & $\begin{array}{l}\text { Presents the first individual of } \\
\text { a role individual }\end{array}$ & $\begin{array}{l}\text { CIFK - foreign key on CIndividual } \\
\text { RIFK - foreign key on RIndividual }\end{array}$ \\
\hline Successor & $\begin{array}{l}\text { Presents the second individual } \\
\text { of a role individual }\end{array}$ & $\begin{array}{l}\text { CIFK - foreign key on CIndividual } \\
\text { RIFK - foreign key on RIndividual }\end{array}$ \\
\hline $\begin{array}{l}\text { Concept } \\
\text { Nesting }\end{array}$ & $\begin{array}{l}\text { Represents the concept inclu- } \\
\text { sion (hierarchy) axiom }\end{array}$ & $\begin{array}{l}\text { CInFK - foreign key on Concept («child») } \\
\text { COutFK - foreign key on Concept («parent») }\end{array}$ \\
\hline $\begin{array}{l}\text { Concept } \\
\text { Equivalence }\end{array}$ & $\begin{array}{l}\text { Represents the concept equiva- } \\
\text { lence axiom }\end{array}$ & $\begin{array}{l}\text { CForFK - foreign key on Concept («which is equal») } \\
\text { CIsFK - foreign key on Concept («equals to which») }\end{array}$ \\
\hline $\begin{array}{l}\text { Role } \\
\text { Nesting }\end{array}$ & $\begin{array}{l}\text { Represents the role inclusion } \\
\text { (hierarchy) axiom }\end{array}$ & $\begin{array}{l}\text { RInFK - foreign key on Role («child») } \\
\text { ROutFK - foreign key on Role («parent») }\end{array}$ \\
\hline $\begin{array}{l}\text { Role } \\
\text { Equivalence }\end{array}$ & $\begin{array}{l}\text { Represents the role equiva- } \\
\text { lence axiom }\end{array}$ & $\begin{array}{l}\text { RForFK - foreign key on Role («which is equal ») } \\
\text { RIsFK - foreign key on Role («equals to which ») }\end{array}$ \\
\hline CIEquivalence & $\begin{array}{l}\text { Represents the individual } \\
\text { equivalence axiom }\end{array}$ & $\begin{array}{l}\text { CIForFK - foreign key on CIndividual («which is } \\
\text { equal ») } \\
\text { CIIsFK - foreign key on CIndividual («equals to } \\
\text { which ») }\end{array}$ \\
\hline
\end{tabular}

where

Interpretation is a pair $\mathrm{I}=\left(\Delta,{ }^{\bullet}\right)$,

- $\Delta-$ a non-empty set called the domain of interpretation,

- $\cdot$ I - interpretation function.

Interpreter function assigns each atomic concept $\mathrm{A}$ a set $\mathrm{A}^{\mathrm{I}} \subseteq \Delta$, and each atomic role $\mathrm{R}$ a binary relationship $\mathrm{R}^{\mathrm{I}} \subseteq \Delta \times \Delta$.

Further, $\mathrm{C}_{\mathrm{RM}^{2}}^{\mathrm{E}}$ will denote the $\mathrm{RM}^{2}$ relationship extensional. This relationship corresponds to the interpretation of an arbitrary concept $\mathrm{C}$.

Table 4 shows the DL ALC syntax and semantics, as well as their corresponding mapping formulas. 
ALC syntax and semantics and their corresponding mapping formulas

Table 4

\begin{tabular}{|c|c|c|}
\hline Syntax & Semantics & Mapping \\
\hline 1 & 2 & 3 \\
\hline \multicolumn{3}{|r|}{ ALC Syntax Concepts } \\
\hline $\mathrm{T}$ & $\mathrm{T}^{\mathrm{I}}=\Delta$ & $\mathrm{T}_{\mathrm{RM}^{2}}^{\mathrm{E}}=\pi_{\mathrm{Name}}($ CIndividual $)$ \\
\hline$\perp$ & $\perp^{\mathrm{I}}=\emptyset$ & Empty(Name) \\
\hline $\mathrm{C}$ & $\mathrm{C}^{\mathrm{I}} \subseteq \Delta^{\mathrm{I}}$ & $\begin{array}{l}\mathrm{C}_{\mathrm{RM}^{2}}^{\mathrm{E}}= \\
=\pi_{\mathrm{CIndividual.Name}}\left(\sigma_{\text {Concept.Name }={ }^{\prime} \mathrm{C}^{\prime}}\right. \\
\left.\left(\text { CIndividual } \bowtie_{\mathrm{CIPK}=\mathrm{CIFK}}\left(\text { LinkCI } \bowtie_{\mathrm{CFK}=\mathrm{CPK}} \text { Concept }\right)\right)\right)\end{array}$ \\
\hline $\mathrm{R}$ & $\mathrm{R}^{\mathrm{I}} \subseteq \Delta^{\mathrm{I}} \times \Delta^{\mathrm{I}}$ & $\begin{aligned} & \mathrm{R}_{\mathrm{RM}}^{\mathrm{E}}=\pi_{\text {First,Second }}\left(\rho_{\text {CIdividual.Name } / \text { Second }}(\text { CIndividual }\right. \\
& \bowtie_{\mathrm{CIPK}=\mathrm{CFPK}}(\text { Successor } \\
& \bowtie_{\mathrm{RIFK}=\mathrm{RIPK}}\left(\rho_{\text {CIndividual.Name } / \text { First }}(\text { CIndividual }\right. \\
& \bowtie_{\mathrm{CIPK}=\mathrm{CIFK}}(\text { Predecessor } \\
& \bowtie_{\mathrm{RIFK}=\mathrm{RIPK}}\left(\sigma_{\text {Role.Name='R' }}(\text { RIndividual }\right. \\
&\left.\left.\left.\left.\left.\left.\left.\bowtie_{\mathrm{RIPK}=\mathrm{RIFK}}\left(\text { LinkRRI } \bowtie_{\mathrm{RFK}=\mathrm{RPK}} \text { Role }\right)\right)\right)\right)\right)\right)\right)\right)\end{aligned}$ \\
\hline$\neg \mathrm{C}$ & $\Delta^{\mathrm{I}} \mathrm{C}^{\mathrm{I}}$ & $(\neg \mathrm{C})_{\mathrm{RM}^{2}}^{\mathrm{E}}=\pi_{\mathrm{Name}}($ CIndividual $)-\mathrm{C}_{\mathrm{RM}^{2}}^{\mathrm{E}}$ \\
\hline $\mathrm{C} \sqcap \mathrm{D}$ & $(C \sqcap D)^{I}=C^{I} \cap D^{I}$ & $(C \sqcap \mathrm{D})=\mathrm{C}_{\mathrm{RM}^{2}}^{\mathrm{E}} \cap \mathrm{D}_{\mathrm{RM}^{2}}^{\mathrm{E}}$ \\
\hline $\mathrm{C} \sqcup \mathrm{D}$ & $(C \sqcup D)^{I}=C^{I} \cup D^{I}$ & $(C \sqcup D)=C_{\mathrm{RM}^{2}}^{E} \cup D_{\mathrm{RM}^{2}}^{\mathrm{E}}$ \\
\hline$\exists$ R.C & $\begin{array}{l}\exists R . C=\{a \in \Delta \mid \exists b \\
\in \Delta\left((a, b) \in R^{I} \wedge b\right. \\
\left.\left.\in C^{I}\right)\right\}\end{array}$ & $(\exists R . C)_{\mathrm{RM}^{2}}^{\mathrm{E}}=\pi_{\text {First }}\left(\mathrm{R}_{\mathrm{RM}}^{\mathrm{E}} \bowtie_{\text {Second }=\text { Name }} C_{\mathrm{RM}^{2}}^{\mathrm{E}}\right)$ \\
\hline$\forall$ R.C & $\begin{array}{l}\forall R . C=\{a \in \Delta \mid \forall b \\
\in \Delta\left((a, b) \in R^{I} \rightarrow b\right. \\
\left.\left.\in C^{I}\right)\right\}\end{array}$ & $\begin{aligned}(\forall \text { R. C })_{\mathrm{RM}^{2}}^{\mathrm{E}}= & \pi_{\mathrm{First}}\left(\mathrm{R}_{\mathrm{RM}}^{\mathrm{E}}\right)-\pi_{\mathrm{First}}\left(\mathrm{R}_{\mathrm{RM}}^{\mathrm{E}} \cap\left(\pi_{\mathrm{First}}\left(\mathrm{R}_{\mathrm{RM}}^{\mathrm{E}}\right)\right.\right. \\
& \left.\left.\times\left(\pi_{\text {Second }}\left(\mathrm{R}_{\mathrm{RM}^{2}}^{\mathrm{E}}\right)-\pi_{\mathrm{Name}}\left(\mathrm{C}_{\mathrm{RM}^{2}}^{\mathrm{E}}\right)\right)\right)\right)\end{aligned}$ \\
\hline \multicolumn{3}{|r|}{ Number restrictions, nominals } \\
\hline$(\geq \mathrm{nR})$ & $\begin{array}{c}(\geq n R)^{I}=\{e \in \Delta \mid \\
\left.\left|R^{I}(e)\right| \geq n\right\}\end{array}$ & $\begin{array}{l}(\geq \mathrm{nR})_{\mathrm{RM}}^{\mathrm{E}}=\pi_{\mathrm{First}}\left(\underset{1 \leq i<j \leq \mathrm{n}}{\sigma} \mathrm{R}_{\mathrm{i}} \cdot \operatorname{Second}_{\mathrm{i}} \neq \mathrm{R}_{\mathrm{j}} \cdot \operatorname{Second}_{\mathrm{j}}\left(\begin{array}{c}* \\
1 \leq i \leq n\end{array}\right.\right. \\
\left.\rho_{\mathrm{R}_{\mathrm{i}}\left({\text { First }, S^{2} \text { econd }}_{\mathrm{i}}\right)}\left(\mathrm{R}_{\mathrm{RM}^{2}}^{\mathrm{E}}\right)\right)\end{array}$ \\
\hline$(\leq \mathrm{nR})$ & $\begin{array}{r}(\leq n R)^{I}=\{e \in \Delta \mid \\
\left.\quad\left|R^{I}(e)\right| \leq n\right\}\end{array}$ & $\begin{array}{l}(\leq n R)_{\mathrm{RM}^{2}}^{\mathrm{E}}=\pi_{\text {Name }}(\text { Cindividual })-\pi_{\text {First }}\left(\underset{1 \leq i<j \leq n+1}{\sigma} \mathrm{R}_{\mathrm{i}} \cdot \text { Second }_{\mathrm{i}} \neq\right. \\
\left.\mathrm{R}_{\mathrm{j}} \cdot \text { Second }_{\mathrm{j}}\left(\underset{1 \leq \mathrm{i} \leq \mathrm{n}+1}{*} \rho_{\mathrm{R}_{\mathrm{i}}\left(\text { First Second }_{\mathrm{i}}\right)}\left(\mathrm{R}_{\mathrm{RM}^{2}}^{\mathrm{E}}\right)\right)\right)\end{array}$ \\
\hline$(\geq \mathrm{nR} \cdot \mathrm{C})$ & $\begin{array}{l}(\geq \mathrm{nR} \cdot \mathrm{C})^{\mathrm{I}}=\{\mathrm{e} \in \Delta \mid \\
\left.\quad\left|\mathrm{R}^{\mathrm{I}}(\mathrm{e}) \cap \mathrm{C}^{\mathrm{I}}\right| \geq \mathrm{n}\right\}\end{array}$ & $\begin{array}{l}(\geq \mathrm{nR} . \mathrm{C})_{\mathrm{RM}}^{\mathrm{E}}=\pi_{\text {First }}\left(\underset{1 \leq i<j \leq \mathrm{n}}{\sigma} \mathrm{R}_{\mathrm{i}} \cdot \text { Second }_{\mathrm{i}} \neq \mathrm{R}_{\mathrm{j}} \cdot \operatorname{Second}_{\mathrm{j}}(\underset{1 \leq i \leq n}{*}\right. \\
\left.\left.\left.\rho_{\mathrm{R}_{\mathrm{i}} \text { (First,Second }}\right)\left(\mathrm{R}_{\mathrm{RM}^{2}}^{\mathrm{E}} \bowtie_{\text {Second }=\text { Name }} C_{\mathrm{RM}^{2}}^{2}\right)\right)\right)\end{array}$ \\
\hline$(\leq \mathrm{nR} \cdot \mathrm{C})$ & $\begin{array}{l}(\leq \mathrm{nR} \cdot \mathrm{C})^{\mathrm{I}}=\{\mathrm{e} \in \Delta \mid \\
\left.\left|\mathrm{R}^{\mathrm{I}}(\mathrm{e}) \cap \mathrm{C}^{\mathrm{I}}\right| \leq \mathrm{n}\right\}\end{array}$ & $\begin{array}{l}(\leq \mathrm{nR} \cdot \mathrm{C})_{\mathrm{RM}^{2}}^{\mathrm{E}}=\pi_{\text {Name }}(\text { CIndividual })-\pi_{\text {First }}\left(\underset{1 \leq i<j \leq \mathrm{n}}{\sigma} \mathrm{R}_{\mathrm{i}} \cdot \text { Second }_{\mathrm{i}} \neq\right. \\
\mathrm{R}_{\mathrm{j}} \text {. Second } \\
\left.\left.\text { Second }_{1 \leq i \leq n}^{*} \rho_{\mathrm{R}_{\mathrm{i}}\left(\text { First,Second }_{\mathrm{i}}\right)}\left(\mathrm{R}_{\mathrm{RM}^{2}}^{\mathrm{E}} \bowtie_{\text {Second=Name }} C_{\mathrm{RM}^{2}}^{\mathrm{E}}\right)\right)\right)\end{array}$ \\
\hline$\{a\}$ & $\{a\}^{\mathrm{I}}$ & $\{\mathrm{a}\}_{\mathrm{RM}^{2}}^{\mathrm{E}}=\left\{\mathrm{a}_{\mathrm{RM}^{2}}^{\mathrm{E}}\right\}$ \\
\hline \multicolumn{3}{|r|}{ Role Constructors } \\
\hline $\mathrm{R}^{-}$ & $\begin{array}{l}\left(\mathrm{R}^{-}\right)^{\mathrm{I}}=\{(\mathrm{e}, \mathrm{d}) \\
\left.\in \Delta \times \Delta \mid(\mathrm{d}, \mathrm{e}) \in \mathrm{R}^{\mathrm{I}}\right\}\end{array}$ & $\left(\mathrm{R}^{-}\right)_{\mathrm{RM}^{2}}^{\mathrm{E}}=\left(\rho_{\mathrm{R}(\text { Second,First })}\left(\mathrm{R}_{\mathrm{RM}^{2}}^{\mathrm{E}}\right)\right)$ \\
\hline
\end{tabular}




\begin{tabular}{|c|c|c|}
\hline 1 & 2 & 3 \\
\hline$\neg \mathrm{R}$ & $(\neg \mathrm{R})^{\mathrm{I}}=\Delta \times \Delta \backslash \mathrm{R}^{\mathrm{I}}$ & $\begin{aligned}(\neg \mathrm{R})_{\mathrm{RM}}^{\mathrm{E}}= & \left(\rho_{\text {Name } / \text { First }}\left(\pi_{\text {Name }}(\text { CIndividual })\right)\right. \\
& \left.\times \rho_{\text {Name } / \text { Second }}\left(\pi_{\text {Name }}(\text { CIndividual })\right)\right)-\mathrm{R}_{\mathrm{RM}^{2}}^{\mathrm{E}}\end{aligned}$ \\
\hline $\mathrm{R} \sqcap \mathrm{S}$ & $(\mathrm{R} \cap \mathrm{S})^{\mathrm{I}}=\mathrm{R}^{\mathrm{I}} \cap \mathrm{S}^{\mathrm{I}}$ & $(\mathrm{R} \sqcap \mathrm{S})_{\mathrm{RM}^{2}}^{\mathrm{E}}=\mathrm{R}_{\mathrm{RM}^{2}}^{\mathrm{E}} \cap \mathrm{S}_{\mathrm{RM}^{2}}^{\mathrm{E}}$ \\
\hline $\mathrm{R} \sqcup \mathrm{S}$ & $(R \sqcup S)^{I}=R^{I} \cup S^{I}$ & $(\mathrm{R} \sqcup \mathrm{S})_{\mathrm{RM}^{2}}^{\mathrm{E}}=\mathrm{R}_{\mathrm{RM}^{2}}^{\mathrm{E}} \cup \mathrm{S}_{\mathrm{RM}^{2}}^{\mathrm{E}}$ \\
\hline $\mathrm{R} \circ \mathrm{S}$ & $\begin{array}{l}\left(\mathrm{R}^{\circ} \mathrm{S}\right)^{\mathrm{I}}=\{(\mathrm{e}, \mathrm{d}) \in \\
\Delta \times \Delta \mid \exists \mathrm{c} \in \Delta((\mathrm{e}, \mathrm{c}) \\
\left.\left.\in \mathrm{R}^{\mathrm{I}} \wedge(\mathrm{c}, \mathrm{d}) \in \mathrm{S}^{\mathrm{I}}\right)\right\}\end{array}$ & $(\mathrm{R} \circ \mathrm{S})_{\mathrm{RM}^{2}}^{\mathrm{E}}=\pi_{\mathrm{R} . \text { First } \text { S.Second }}\left(\mathrm{R}_{\mathrm{RM}^{2}}^{\mathrm{E}} \bowtie_{\mathrm{R} . S e c o n d=S . F i r s t} \mathrm{~S}_{\mathrm{RM}^{2}}^{\mathrm{E}}\right)$ \\
\hline $\operatorname{id}(C)$ & $\begin{array}{l}(\operatorname{id}(C))^{I}=\{(e, e) \\
\left.\quad \in \Delta \times \Delta \mid e \in C^{I}\right\}\end{array}$ & $\begin{aligned}(\operatorname{id}(C))_{\mathrm{RM}^{2}}^{\mathrm{E}}= & \left(\rho_{\text {Name } / \text { First }}\left(\pi_{\mathrm{Name}} \mathrm{C}_{\mathrm{RM}^{2}}^{\mathrm{E}}\right)\right) \\
& \bowtie_{\text {First }=\text { Second }}\left(\rho_{\text {Name } / \text { Second }}\left(\pi_{\mathrm{C} . \mathrm{Name}} \mathrm{C}_{\mathrm{RM}}^{\mathrm{E}}\right)\right)\end{aligned}$ \\
\hline $\mathrm{R}^{+}$ & $\left(R^{+}\right)^{I}=\bigcup_{n \geq 1}\left(R^{I}\right)^{n}$ & $\left(\mathrm{R}^{+}\right)_{\mathrm{RM}^{2}}^{\mathrm{E}}=\left(\mathrm{R}_{\mathrm{RM}^{2}}^{\mathrm{E}}\right)^{+}$ \\
\hline $\mathrm{R}^{*}$ & $\left(R^{*}\right)^{I}=\bigcup_{n \geq 0}\left(R^{I}\right)^{n}$ & $\begin{array}{l}\left(\mathrm{R}^{*}\right)_{\mathrm{RM}}^{\mathrm{E}} \\
=\left(\rho_{\text {Name } / \text { First }}\left(\pi_{\text {Name }} \text { CIndividual }\right)\right) \\
\bowtie_{\text {First }=\text { Second }}\left(\rho_{\text {Name } / \text { Second }}\left(\pi_{\text {Name }} \text { CIndividual }\right)\right) \cup\left(\mathrm{R}^{+}\right)_{\mathrm{RM}^{2}}^{\mathrm{E}}\end{array}$ \\
\hline
\end{tabular}

The DL axiom mapping has been shown in the conceptual ER scheme. Each axiom has its own entity. Each axiom has its own binary relationship in $\mathrm{RM}^{2}$. Each relationship has two foreign keys. Each of the keys refers to concepts, roles or individuals about which the axiom is formulated.

ConceptNesting (CInFK, COutFK)

$\mathrm{C} \sqsubseteq \mathrm{D}$

ConceptEquivalence (CForFK, CIsFK)

$\mathrm{C} \equiv \mathrm{D}$

RoleNesting (RInFK, ROutFK)

$\mathrm{R} \sqsubseteq \mathrm{S}$

RoleEquivalence (RForFK, RIsFK)

$\mathrm{R} \equiv \mathrm{S}$

CIEquivalence (CIForFK, CIIsFK)

$\mathrm{a}=\mathrm{b}$

Role(RPK, Name IsTransitive)

$\mathrm{TR}(\mathrm{R})$

\section{Mapping $\mathbf{R M}^{2}$ into RDF}

$\mathrm{RM}^{2}$ to RDF mappings can be meaningfully divided into several parts. Firstly, how to transform each RDB relationship of an $\mathrm{RM}^{2}$ will be shown. All RDB relationships can be divided into the following groups: basic relationships (concepts, roles, individuals), connective relationships and axiom relationships. The mapping of all ALC constructs will be shown next. The description of map- ping mechanisms for number restrictions, roles restrictions and nominals completes this section.

Since empty relationships map to an empty RDF graph, there are a number of rows in each relationship to be an example. These strings will be mapped to the RDF triples using R2R ML triple maps. These triple maps are the mechanism for mapping RDB relationships into RDF.

Examples of mapping are present for only one row of each logical table. This is done to save space and to emphasize the rules themselves, not just their use.

Turtle syntax was used to describe triple maps, as well as the following notation:

@prefix rr: $<$ http://www.w3.org/ns/r2rml\#>

@prefix ex: http://example.com/Ch\#

\section{Basic concepts}

\subsection{Concept}

SQL table Concept

\begin{tabular}{|l|l|}
\hline CPK & Name \\
\hline 1 & C \\
\hline 2 & D \\
\hline 3 & E \\
\hline 4 & F \\
\hline
\end{tabular}

R2R ML Triple Map

<\#TriplesMap1> 
rr:logicalTable [ rr:tableName «Concept»]; r:subjectMap [ rr:template

«http://example.com/Ch\#/Concept/ $\{\mathrm{CPK}\} »$; rr:class ex:Concept;

] rr:predicateObjectMap [ rr:predicate ex:name; rr:objectMap [rr:column «Name»]; ]

RDF output example

<http://example.com/Ch\#/Concept/l>rdf:type ex:Concept.

$<$ http://example.com/Ch\#/Concept/1>ex:name "C

RDF output example graph

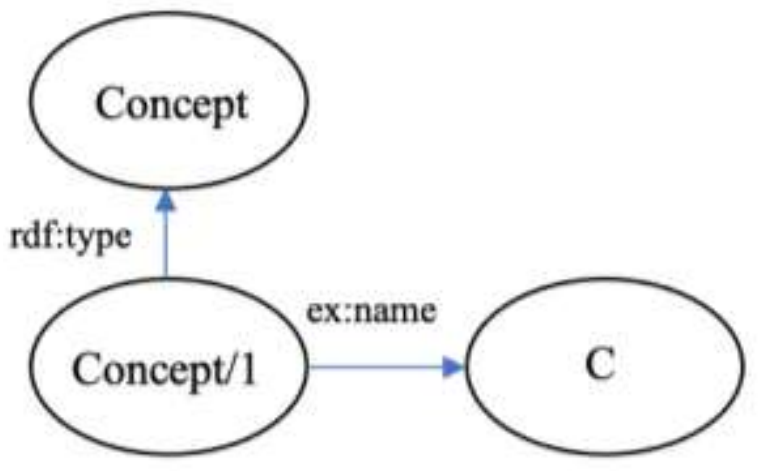

1.2. Role

SQL table Role

\begin{tabular}{|l|l|l|}
\hline RPK & Name & IsTransitive \\
\hline 56 & R & No \\
\hline 67 & S & No \\
\hline 89 & T & No \\
\hline 34 & Z & No \\
\hline 23 & U & Yes \\
\hline
\end{tabular}

$\underline{\text { R2R ML Triple Map }}$

<\#TriplesMap2>

rr:logicalTable [ rr:tableName «Role»];

r:subjectMap [

rr:template

«http://example.com/Ch\#/Role/ $\{$ RPK $\} » ;$

rr:class ex:Role;

]

rr:predicateObjectMap [

rr:predicate ex:name;

rr:objectMap [rr:column «Name»];

]

rr:predicateObjectMap [

rr:predicate ex:IsTransitive;
rr:objectMap [rr:column «IsTransitive»];

]

RDF output example

<http://example.com/Ch\#/Role/56> rdf:type ex:Role.

$<$ http://example.com/Ch\#/Role/56> ex:name " $R$ ". <http://example.com/Ch\#/Role/56>

ex:IsTransitive "No".

RDF output example graph

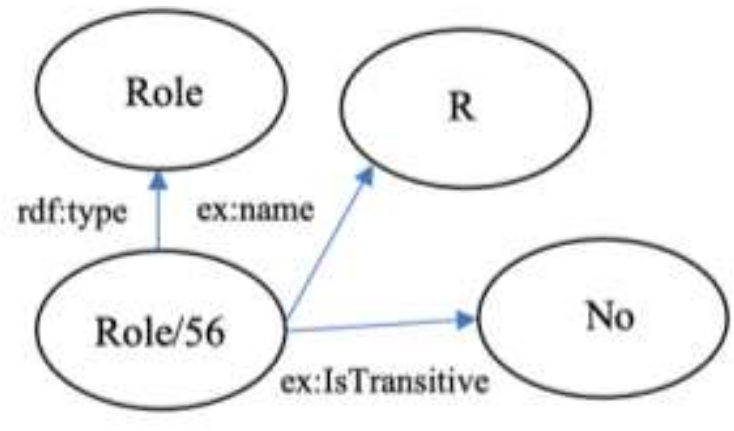

\subsection{CIndividual}

SQL table CIndividual

\begin{tabular}{|l|l|}
\hline CIPK & Name \\
\hline 100 & abc \\
\hline 101 & def \\
\hline 102 & aaa \\
\hline 103 & bbb \\
\hline 104 & ccc \\
\hline 105 & ddd \\
\hline
\end{tabular}

$\underline{\text { R2R ML Triple Map }}$

<\#TriplesMap3>

rr:logicalTable [ rr:tableName «CIndividual»];

r:subjectMap [

rr:template

«http://example.com/Ch\#/CIndividual/ $\{\mathrm{CIPK}\} »$;

rr:class ex:CIndividual;

]

rr:predicateObjectMap [

rr:predicate ex:name;

rr:objectMap [rr:column «Name»];

]

RDF output example

<http://example.com/Ch\#/CIndividual/100> rdf:type ex:CIndividual.

<http://example.com/Ch\#/CIndividual/100>

ex:name "abc". 


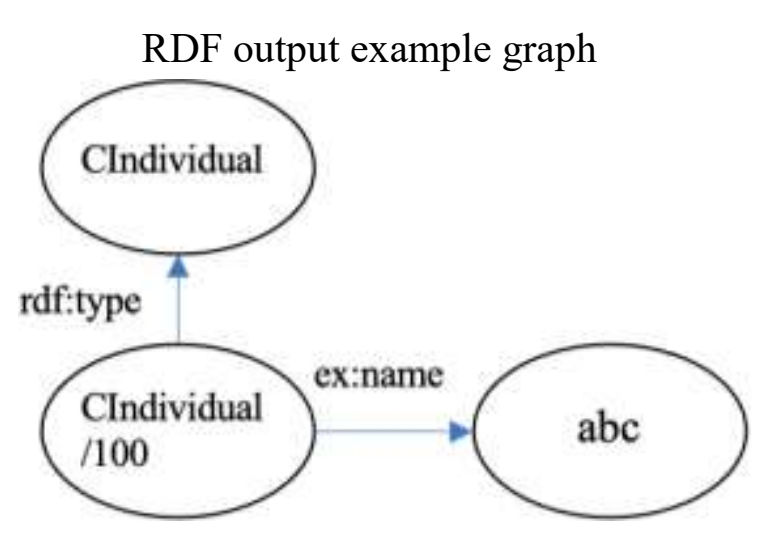

\subsection{RIndividual}

SQL table RIndividual

\begin{tabular}{|c|}
\hline RIPK \\
\hline 10 \\
\hline 11 \\
\hline 12 \\
\hline 13 \\
\hline 14 \\
\hline 15 \\
\hline
\end{tabular}

\section{$\underline{\text { R2R ML Triple Map }}$}

<\#TriplesMap4>

rr:logicalTable [ rr:tableName «RIndividual»];

r:subjectMap [

rr:template

«http://example.com/Ch\#/RIndividual/ $\{$ RIPK $\} » ;$ rr:class ex:RIndividual;

]

rr:predicateObjectMap [

rr:predicate ex:RIPK;

rr:objectMap [rr:column «RIPK»];

]

RDF output example

<http://example.com/Ch\#/RIndividual/10>

rdf:type ex:RIndividual.

<http://example.com/Ch\#/RIndividual/10> ex:RIPK 10.

RDF output example graph

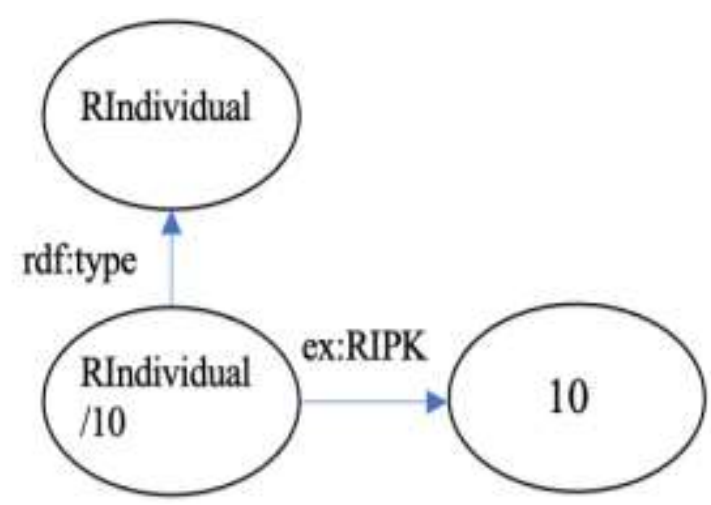

\section{Relationship-bundles}

\subsection{Domain}

SQL table Domain

\begin{tabular}{|l|l|}
\hline CFK & RFK \\
\hline 1 & 56 \\
\hline 2 & 67 \\
\hline
\end{tabular}

$\underline{\text { R2R ML Triple Map }}$

$<\#$ TriplesMap5>

rr:logicalTable [ rr:tableName «Domain»];

r:subjectMap [

rr:template

«http://example.com/Ch\#//Domain/\{CFK\}; RFK

$\xi » ;$

rr:class ex:Domain;

]

rr:predicateObjectMap [

rr:predicate ex:CFK

rr:objectMap [

a rr:RefObjectMap ;

rr:parentTriplesMap <\#TriplesMap1>

rr:joinCondition [

rr:child «CFK»;

rr:parent «CPK»;

]]]

rr:predicateObjectMap [

rr:predicate ex:RFK

rr:objectMap [

a rr:RefObjectMap ;

rr:parentTriplesMap <\#TriplesMap2>

rr:joinCondition [

rr:child «RFK»;

rr:parent «RPK»;

]]]

RDF output example

$<$ http://example.com/Ch\#/Domain/1;56>rdf:type ex:Domain.

$<$ http://example.com/Ch\#/Domain/1;56>ex:CFK

$<$ http://example.com/Ch\#/Concept/1>.

$<$ http://example.com/Ch\#/Domain/1;56>ex:RFK

$<$ http://example.com/Ch\#/Role/56>. 


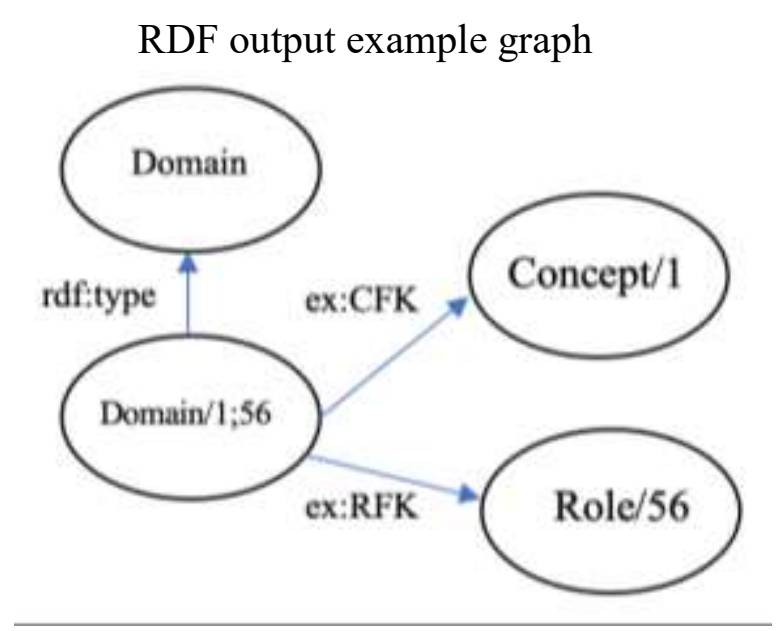

\subsection{Range}

SQL table Range

\begin{tabular}{|l|l|}
\hline CFK & RFK \\
\hline 2 & 56 \\
\hline 1 & 67 \\
\hline
\end{tabular}

\section{$\underline{\text { R2R ML Triple Map }}$}

$<\#$ TriplesMap6>

rr:logicalTable [ rr:tableName «Range»];

r:subjectMap [

rr:template

«http://example.com/Ch\#/Range/ $\{\mathrm{CFK}\} ;\{\mathrm{RFK}\} » ;$ rr:class ex:Range;

]

rr:predicateObjectMap [

rr:predicate ex:CFK

rr:objectMap [

a rr:RefObjectMap ;

rr:parentTriplesMap <\#TriplesMap1>

rr:joinCondition [ rr:child «CFK»;

rr:parent $« \mathrm{CPK} »$;

]]]

rr:predicateObjectMap [

rr:predicate ex:RFK

rr:objectMap [

a rr:RefObjectMap ;

rr:parentTriplesMap <\#TriplesMap2>

rr:joinCondition [

rr:child «RFK»;

rr:parent $« R P K »$;

]]]

RDF output example

$<$ http://example.com/Ch\#/Range/2;67>rdf:type ex:Range.
$<$ http://example.com/Ch\#/Range/2;67>ex:CFK

$<$ http://example.com/Ch\#/Concept/2>.

$<$ http://example.com/Ch\#/Range/2;67>ex:RFK

$<$ http://example.com/Ch\#/Role/67>.

RDF output example graph

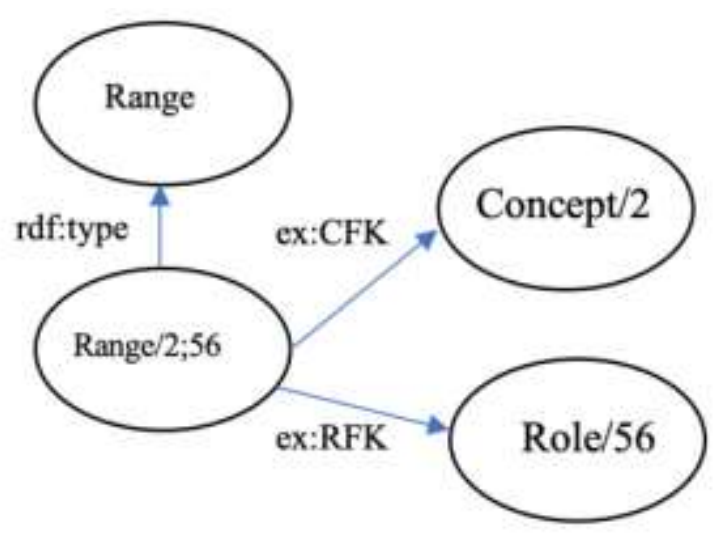

2.3. LinkCI

SQL table LinkCI

\begin{tabular}{|l|l|}
\hline CFK & CIFK \\
\hline 1 & 100 \\
\hline 1 & 101 \\
\hline 2 & 102 \\
\hline 2 & 103 \\
\hline 2 & 104 \\
\hline 2 & 101 \\
\hline
\end{tabular}

$\underline{\text { R2R ML Triple Map }}$

$<$ \#TriplesMap7>

rr:logicalTable [ rr:tableName «LinkCI»];

r:subjectMap [

rr:template

«http://example.com/Ch\#/LinkCI/\{CFK $\} ;\{$ CIFK $\}$ $»$;

rr:class ex:LinkCI;

]

rr:predicateObjectMap [

rr:predicate ex:CFK

rr:objectMap [

a rr:RefObjectMap ;

rr:parentTriplesMap <\#TriplesMap1>

rr:joinCondition [ rr:child «CFK»; rr:parent «CPK»;

]]]

rr:predicateObjectMap [

rr:predicate ex:CIFK

rr:objectMap [ 
a rr:RefObjectMap ;

rr:parentTriplesMap <\#TriplesMap3>

rr:joinCondition [

rr:child «CIFK»;

rr:parent «CIPK»;

]]]

RDF output example

$<$ http://example.com/Ch\#/LinkCI/1;100>rdf:type ex:LinkCI.

$<$ http://example.com/Ch\#/LinkCI/1;100>ex:CFK

$<$ http://example.com/Ch\#/Concept/1>.

$<$ http://example.com/Ch\#/LinkCI/1;100>ex:CIFK

$<$ http://example.com/Ch\#/CIndividual/100>.

RDF output example graph

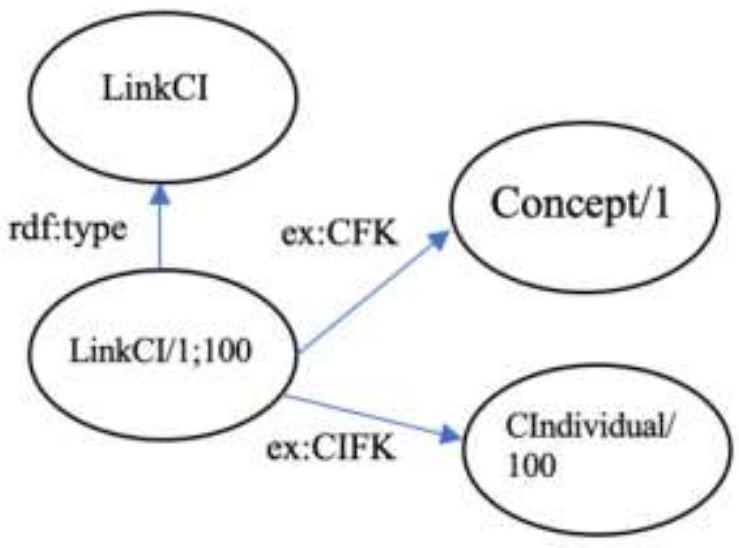

\subsection{LinkRRI}

SQL table LinkRRI

\begin{tabular}{|l|l|}
\hline RFK & RIFK \\
\hline 56 & 10 \\
\hline 56 & 11 \\
\hline 67 & 12 \\
\hline 67 & 13 \\
\hline 67 & 14 \\
\hline 56 & 15 \\
\hline
\end{tabular}

R2R ML Triple Map

$<\#$ TriplesMap8>

rr:logicalTable [ rr:tableName «LinkRRI»];

r:subjectMap [

rr:template

«http://example.com/Ch\#/LinkRRI/\{RFK $\} ;\{$ RIFK

\}»;

rr:class ex:LinkRRI;

]

rr:predicateObjectMap [ rr:predicate ex:RFK

rr:objectMap [

a rr:RefObjectMap ;

rr:parentTriplesMap <\#TriplesMap2>

rr:joinCondition [ rr:child «RFK»; rr:parent «RPK»;

]1]

rr:predicateObjectMap [

rr:predicate ex:RIFK

rr:objectMap [

a rr:RefObjectMap ;

rr:parentTriplesMap <\#TriplesMap4>

rr:joinCondition [

rr:child «RIFK»;

rr:parent $\ll R I P K »$;

]]]

RDF output example

$<$ http://example.com/Ch\#/LinkRRI/56;10> rdf:type ex:LinkRRI.

$<$ http://example.com/Ch\#/LinkRRI/56;10> ex:RFK <http://example.com/Ch\#/Role/56>. $<$ http://example.com/Ch\#/LinkRRI/56;10> ex:RIFK

$<$ http://example.com/Ch\#/RIndividual/10>.

RDF output example graph

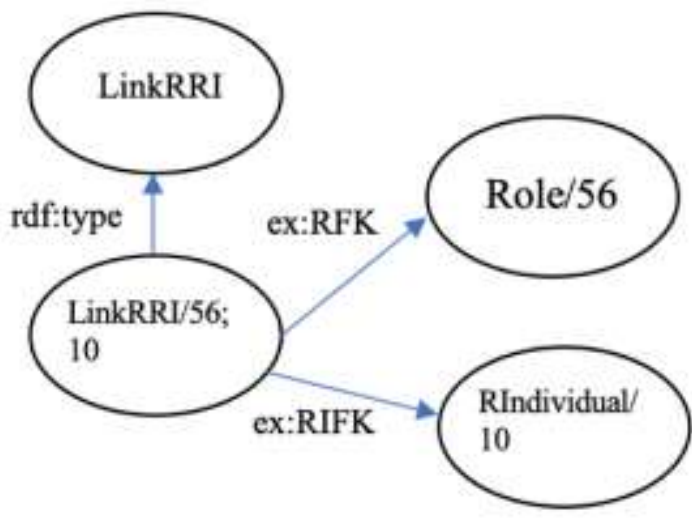

\subsection{Predecessor}

SQL table Predecessor

\begin{tabular}{|l|l|}
\hline CIFK & RIFK \\
\hline 100 & 10 \\
\hline 101 & 11 \\
\hline 100 & 15 \\
\hline 102 & 12 \\
\hline 103 & 13 \\
\hline 104 & 14 \\
\hline
\end{tabular}




\section{$\underline{\text { R2R ML Triple Map }}$}

$<\#$ TriplesMap9>

rr:logicalTable [ rr:tableName «Predecessor»];

r:subjectMap [

rr:template

«http://example.com/Ch\#/Predecessor/ $\{\mathrm{CIFK}\} ;\{\mathrm{R}$

IFK $\} »$

rr:class ex:Predecessor;

]

rr:predicateObjectMap [

rr:predicate ex:CIFK

rr:objectMap [

a rr:RefObjectMap ;

rr:parentTriplesMap <\#TriplesMap3>

rr:joinCondition [

rr:child «CIFK»;

rr:parent «CIPK»;

]]]

rr:predicateObjectMap [

rr:predicate ex:RIFK

rr:objectMap [

a rr:RefObjectMap ;

rr:parentTriplesMap <\#TriplesMap4>

rr:joinCondition [

rr:child $«$ RIFK»;

rr:parent «RIPK»;

]]]

RDF output example

$<$ http://example.com/Ch\#/Predecessor/100;10> rdf:type ex:Predecessor.

$<$ http://example.com/Ch\#/Predecessor/100;10>ex :CIFK

$<$ http://example.com/Ch\#/CIndividual/100>.

$<$ http://example.com/Ch\#/Predecessor/100;10>ex

:RIFK

$<$ http://example.com/Ch\#/RIndividual/10>.

RDF output example graph

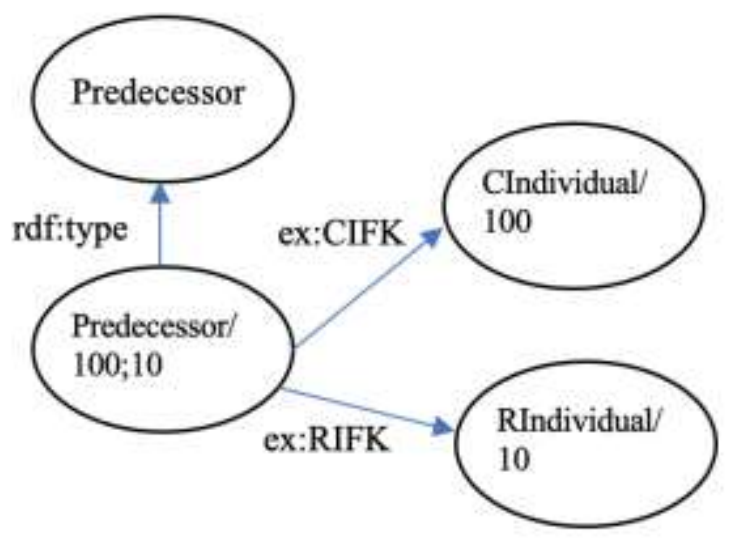

\subsection{Successor}

SQL table Successor

\begin{tabular}{|l|l|}
\hline CIFK & RIFK \\
\hline 102 & 10 \\
\hline 103 & 11 \\
\hline 104 & 15 \\
\hline 100 & 12 \\
\hline 101 & 13 \\
\hline 101 & 14 \\
\hline
\end{tabular}

$\underline{\text { R2R ML Triple Map }}$

$<\#$ TriplesMap10>

rr:logicalTable [ rr:tableName «Successor»];

r:subjectMap [

rr:template

«http://example.com/Ch\#/Successor/ \{CIFK $\} ;\{\mathrm{RI}$

FK $\} »$;

rr:class ex:Successor;

]

rr:predicateObjectMap [

rr:predicate ex:CIFK

rr:objectMap [

a rr:RefObjectMap ;

rr:parentTriplesMap <\#TriplesMap3>

rr:joinCondition [

rr:child «CIFK»;

rr:parent $« \mathrm{CIPK} »$;

]]]

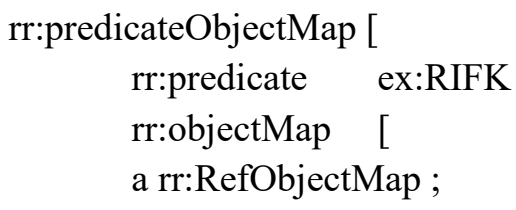

]]]

RDF output example

$<$ http://example.com/Ch\#/Successor/102;10>

rdf:type ex:Successor.

$<$ http://example.com/Ch\#/Successor/102;10>

ex:CIFK

$<$ http://example.com/Ch\#/CIndividual/102>.

$<$ http://example.com/Ch\#/Successor $\quad / 102 ; 10>$

ex:RIFK

$<$ http://example.com/Ch\#/RIndividual/10>. 
RDF output example graph

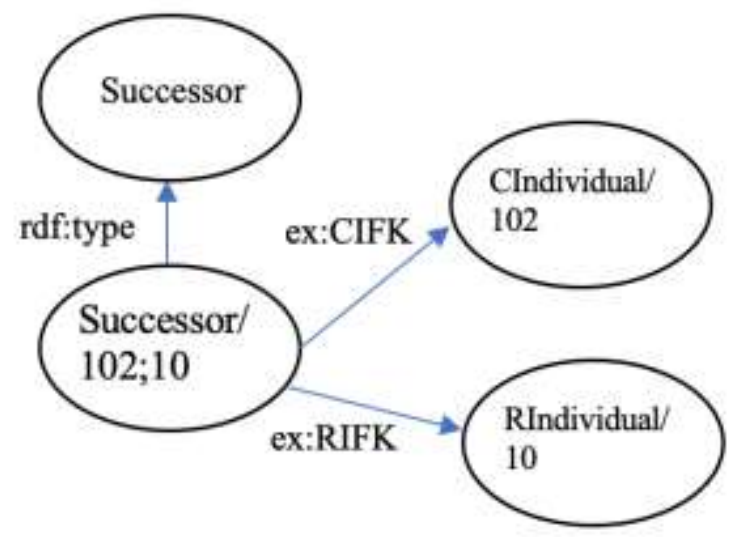

3. Axioms

\subsection{ConceptEquivalence}

SQL table ConceptEquivalence

\begin{tabular}{|l|l|}
\hline CForFK & CIsFK \\
\hline 3 & 4 \\
\hline
\end{tabular}

$\underline{\text { R2R ML Triple Map }}$

<\#TriplesMap11>

rr:logicalTable

[ rr:tableName

«ConceptEquivalence»];

r:subjectMap [

rr:template

«http://data.example.com/ConceptEquivalence/ \{C

ForFK $\} ;\{\mathrm{CIsFK}\} »$;

rr:class ex:ConceptEquivalence;

]

rr:predicateObjectMap [

rr:predicate ex:CForFK

rr:objectMap [

a rr:RefObjectMap ;

rr:parentTriplesMap <\#TriplesMap1>

rr:joinCondition [

rr:child «CForFK»;

rr:parent «CPK»;

]]]

rr:predicateObjectMap [

rr:predicate ex:CIsFK

rr:objectMap [

a rr:RefObjectMap ;

rr:parentTriplesMap <\#TriplesMap1>

rr:joinCondition [

rr:child «CIsFK»;

rr:parent «CPK»;

]]]

RDF output example

$<$ http://example.com/Ch\#/ConceptEquivalence/3,
4> rdf:type ex:ConceptEquivalence.

$<$ http://example.com/Ch\#/ConceptEquivalence/3;

$4>$ ex:CForFK

$<$ http://example.com/Ch\#/Concept/3>.

$<$ http://example.com/Ch\#/ConceptEquivalence/3;

$4>$ ex: $\mathrm{CIs} F \mathrm{~K}$

$<$ http://example.com/Ch\#/Concept/4>

RDF output example graph

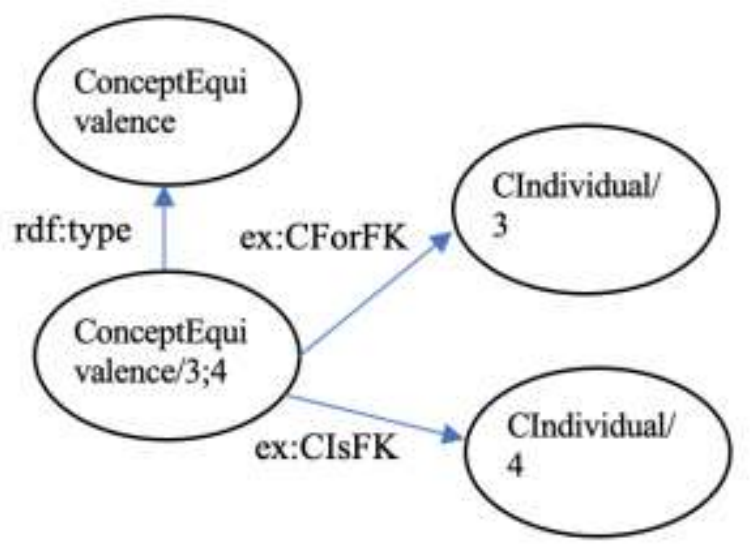

\subsection{ConceptNesting}

SQL table ConceptNesting

\begin{tabular}{|l|l|}
\hline CInFK & COutFK \\
\hline 3 & 1 \\
\hline
\end{tabular}

$\underline{\text { R2R ML Triple Map }}$

$<\#$ TriplesMap12>

rr:logicalTable

[ rr:tableName

«ConceptNesting»];

r:subjectMap [

rr:template

«http://data.example.com/ConceptNesting/ $\{\mathrm{CInF}$

$\mathrm{K}\} ;\{\mathrm{COutFK}\} »$;

rr:class ex:ConceptNesting;

]

rr:predicateObjectMap [

rr:predicate ex:CInFK

rr:objectMap [

a rr:RefObjectMap ;

rr:parentTriplesMap <\#TriplesMap1>

rr:joinCondition [

rr:child «CInFK»;

rr:parent «CPK»;

]]]

rr:predicateObjectMap [

rr:predicate ex:COutFK

rr:objectMap [

a rr:RefObjectMap ; 
rr:parentTriplesMap <\#TriplesMap1> rr:joinCondition [ rr:child «COutFK»; rr:parent «CPK»;

]]]

\section{RDF output example}

<http://example.com/Ch\#/ConceptNesting/3;1> rdf:type ex:ConceptNesting.

$<$ http://example.com/Ch\#/ConceptNesting/3;1>ex :CInFK <http://example.com/Ch\#/Concept/1>. $<$ http://example.com/Ch\#/ConceptNesting/3; $1>$ ex :COutFK <http://example.com/Ch\#/Concept/1>

RDF output example graph

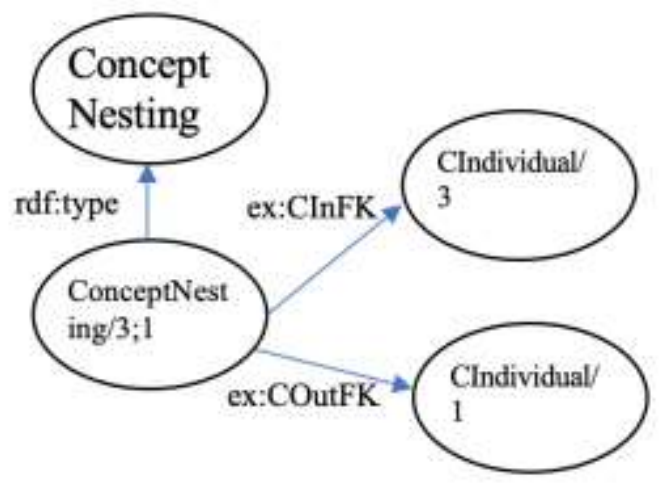

\subsection{RoleNesting}

SQL table RoleNesting

\begin{tabular}{|l|l|}
\hline RInFK & ROutFK \\
\hline 89 & 56 \\
\hline
\end{tabular}

\section{$\underline{\text { R2R ML Triple Map }}$}

$<\#$ TriplesMap13>

rr:logicalTable [rr:tableName «RoleNesting»];

r:subjectMap [

rr:template

«http://example.com/Ch\#/RoleNesting/ $\{$ RInFK $\} ;\{$

ROutFK \}»;

rr:class ex:RoleNesting;

]

rr:predicateObjectMap [

rr:predicate ex:RInFK

rr:objectMap [

a rr:RefObjectMap ;

rr:parentTriplesMap <\#TriplesMap2>

rr:joinCondition [ rr:child «RInFK»; rr:parent $« \mathrm{RPK} »$;

]]]
rr:predicateObjectMap [

rr:predicate ex:ROutFK

rr:objectMap [

a rr:RefObjectMap ;

rr:parentTriplesMap <\#TriplesMap2>

rr:joinCondition [ rr:child «ROutFK»; rr:parent «RPK»;

]]]

RDF output example

<http://example.com/Ch\#/RoleNesting/89;56>

rdf:type ex:RoleNesting.

<http://example.com/Ch\#/RoleNesting/89;56> ex:RInFK

<http://example.com/Ch\#/RoleNesting/89>

<http://example.com/Ch\#/RoleNesting/89;56>

ex:ROutFK

$<$ http://example.com/Ch\#/RoleNesting/56>

RDF output example graph

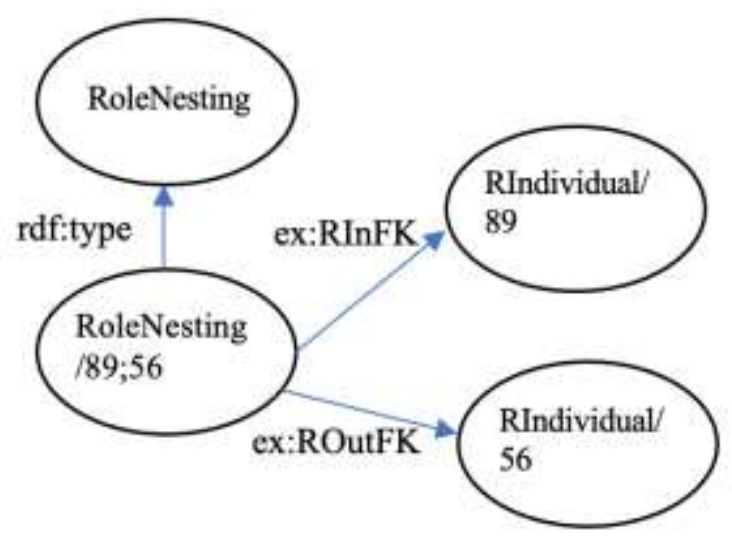

3.4. RoleEquivalence

SQL table RoleEquivalence

\begin{tabular}{|l|l|}
\hline RForFK & RIsFK \\
\hline 89 & 34 \\
\hline
\end{tabular}

$\underline{\text { R2R ML Triple Map }}$

$<$ \#TriplesMap14>

rr:logicalTable

rr:tableName

«RoleEquivalence»];

r:subjectMap [

rr:template

«http://example.com/Ch\#/RoleEquivalence/ \{RFor $\mathrm{FK}\} ;\{\mathrm{RIsFK}\} »$;

rr:class ex:RoleNesting; 
rr:predicateObjectMap [

rr:predicate ex:RForFK

rr:objectMap [

a rr:RefObjectMap ;

rr:parentTriplesMap <\#TriplesMap2>

rr:joinCondition [ rr:child «RForFK»; rr:parent «RPK»;

]]]

rr:predicateObjectMap [

rr:predicate ex:RIsFK

rr:objectMap [

a rr:RefObjectMap ;

rr:parentTriplesMap <\#TriplesMap2>

rr:joinCondition [

rr:child «RIsFK»;

rr:parent «RPK»;

]]

RDF output example

$<$ http://example.com/Ch\#/RoleEquivalence/89;34

$>$ rdf:type ex:RoleEquivalence.

$<$ http://example.com/Ch\#/RoleEquivalence/89;34

>ex:RForFK

$<$ http://example.com/Ch\#/RoleEquivalence/89>

$<$ http://example.com/Ch\#/RoleEquivalence/89;34

$>$ ex:RIsFK

$<$ http://example.com/Ch\#/RoleEquivalence/56>

RDF output example graph

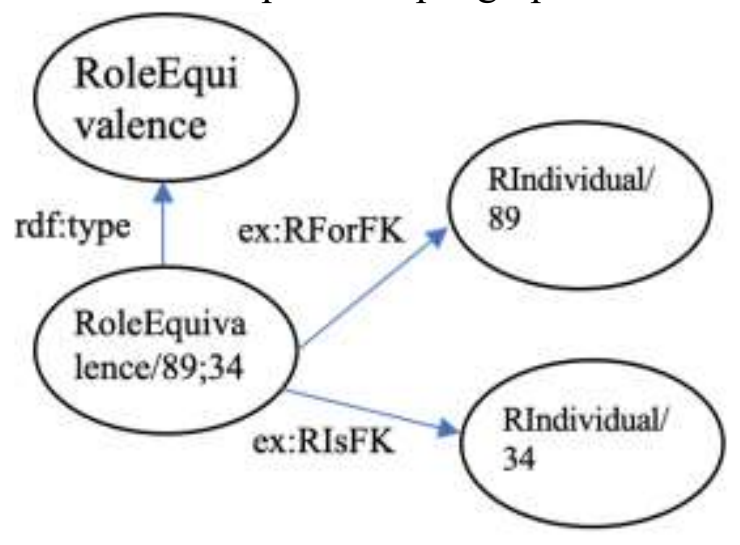

3.5. Transitive role

SQL table Role

\begin{tabular}{|l|l|l|}
\hline RPK & Name & IsTransitive \\
\hline 56 & R & No \\
\hline 67 & S & No \\
\hline 89 & T & No \\
\hline 34 & Z & No \\
\hline 23 & U & Yes \\
\hline
\end{tabular}

<\#TriplesMap15>

$\underline{\text { R2R ML Triple Map }}$

rr:logicalTable [ rr:tableName «Role»];

r:subjectMap [

rr:template

«http://example.com/Ch\#/Role/ $\{\mathrm{RPK}\} »$;

rr:class ex:Role;

]

rr:predicateObjectMap [

rr:predicate ex:IsTransitive;

rr:objectMap [rr:column «IsTransitive»];

]

RDF output example

<http://example.com/Ch\#/Role/23> rdf:type ex:Role.

<http://example.com/Ch\#/Role/23>

ex:IsTransitive "Yes".

RDF output example graph

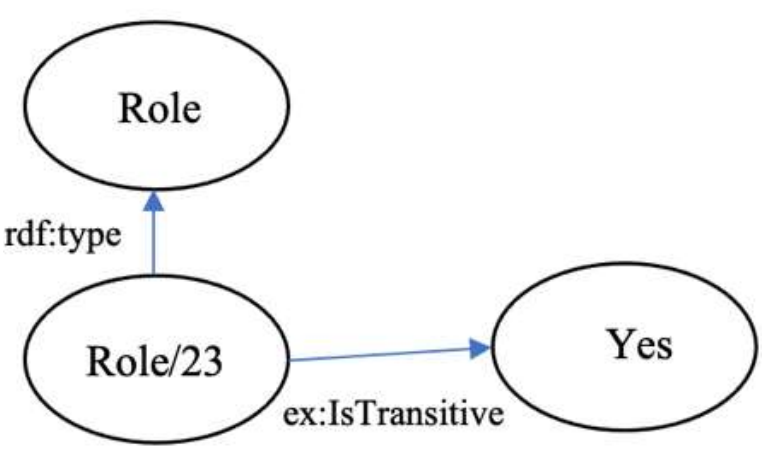

\subsection{CIEquivalence}

SQL table CIEquivalence

\begin{tabular}{|l|l|}
\hline CIForFK & CIIsFK \\
\hline 105 & 104 \\
\hline
\end{tabular}

\section{$\underline{\text { R2R ML Triple Map }}$}

$<\#$ TriplesMap16>

rr:logicalTable [ rr:tableName «CIEquivalence»]; r:subjectMap [

\section{rr:template}

«http://example.com/Ch\#/CIEquivalence/ $\{$ CIForF

$\mathrm{K}\} ;\{\mathrm{CIIsFK}\} »$;

rr:class ex:RoleNesting;

]

rr:predicateObjectMap [

rr:predicate ex:CIForFK

rr:objectMap [

a rr:RefObjectMap ; 
rr:parentTriplesMap $<$ \#TriplesMap3 $>$ rr:joinCondition [ rr:child «CIForFK»; rr:parent «CIPK»;

]]]

rr:predicateObjectMap [

rr:predicate ex:CIIsFK

rr:objectMap [

a rr:RefObjectMap ;

rr:parentTriplesMap <\#TriplesMap3>

rr:joinCondition [

rr:child «CIIsFK»;

rr:parent «CIPK»;

]]]

RDF output example

$<$ http://example.com/Ch\#/CIEquivalence/105;104

$>$ rdf:type ex:CIEquivalence

$<$ http://example.com/Ch\#/CIEquivalence/105;104

$>$ ex:CIForFK

$<$ http://example.com/Ch\#/CIndividual/105>.

$<$ http://example.com/Ch\#/CIEquivalence/105;104

$>$ ex:CIIsFK

$<$ http://example.com/Ch\#/CIndividual/104>.

RDF output example graph

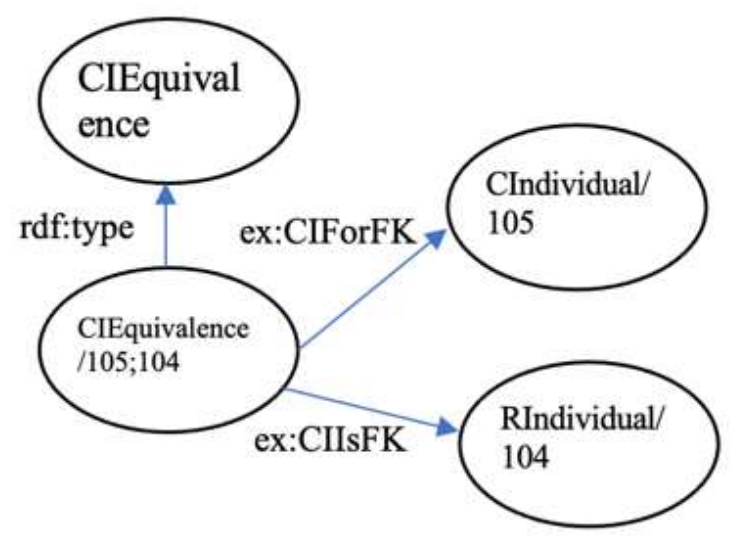

\section{ALC syntax mapping}

\subsection{Concept}

$\mathrm{RA}^{2}$ term

$\mathrm{C}_{\mathrm{RM}^{2}}^{\mathrm{E}}$

$=\pi_{\text {CIndividual.Name }}\left(\sigma_{\text {Concept.Name }={ }^{\prime} \mathrm{C}^{\prime}}(\right.$ CIndividual

$\bowtie_{\mathrm{CIPK}=\mathrm{CIFK}}\left(\right.$ LinkCI $\bowtie_{\mathrm{CFK}=\mathrm{CPK}}$ Concept))

$\underline{\text { R2R ML Triple Map }}$

<\#TriplesMap17>
rr:logicalTable [ rr:sqlQuery

“"”' SELECT ci.Name

FROM CIndividual ci, Concept c, LinkCI lci, WHERE ci.CIPK $=1$ ci.CIFK. AND 1ci.CFK $=$ c.CPK AND c.Name = 'C' '“"'];

r:subjectMap [

rr:template «http://example.com/Ch\#/\{Name\}»; rr:class ex:CIndividual;

]

rr:predicateObjectMap [

rr:predicate ex:name

rr:objectMap [ rr:column: Name;]

]

RDF output example

〈http://example.com/Ch\#/CIndividual/abc> rdf:type ex:CIndividual.

〈http://example.com/Ch\#/CIndividual/abc> ex:name "abc".

RDF output example graph

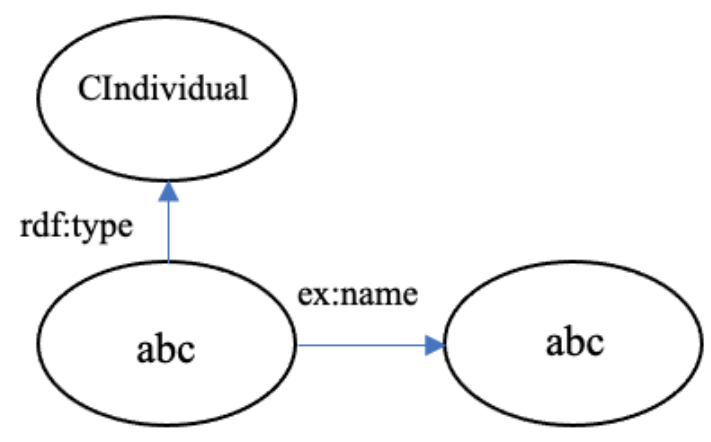

4.2. Role

$\mathrm{RA}^{2}$ term

$\mathrm{R}_{\mathrm{RM}}^{\mathrm{E}}$

$=\pi_{\text {First }, \text { Second }}\left(\rho_{\text {CIdividual.Name.Second }}\right.$ (CIndividual

$\bowtie_{\text {CIPK }=\text { CFPK }}$ (Successor

$\bowtie_{\text {RIFK=RIPK }}$ ( $\rho_{\text {CIndividual.Name.First }}$ (CIndividual

$\bowtie_{\mathrm{CIPK}=\mathrm{CIFK}}$ (Predecessor

$\bowtie_{\text {RIFK=RIPK }}\left(\sigma_{\text {Role.Name }={ }^{\prime}{ }^{\prime}}\right.$ (RIndividual

$\bowtie_{\text {RIPK=RIFK }}($ LinkRRI

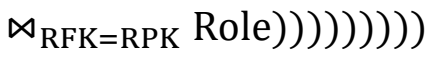

$\underline{\text { R2R ML Triple Map }}$

$<\#$ TriplesMap18>

rr:logicalTable [ rr:sqlQuery

“"” SELECT first.Name AS First, second,Name

AS Second

FROM Role r, RIndividual ri, LinkRRI lrri, Predecessor p, Successor $\mathrm{s}$

CIndividual first, CIndividual.second 
WHERE r.RPK $=$ 1rri.RFK AND 1rri. RIFK $=$ ri.RIPK AND ri.RIPK $=$ p.RIFK AND p.CIFK $=$ first.CIPK AND ri.RIPK = s.RIFK AND s.CIFK = second. CIPK AND r.Name='R" "'"'’];

r:subjectMap [

rr:template

«http://example.com/Ch\#/\{First $\}$ _SSecond $\} »$; rr:class ex:RIndividual;

]

rr:predicateObjectMap [

rr:predicate ex:first

rr:objectMap [ rr:column: First; ]

]

rr:predicateObjectMap [

rr:predicate ex:second

rr:objectMap [ rr:column: Second;

1

RDF output example

$<$ http://example.com/Ch\#/abc_aaa $>$ rdf:type ex:Role;

$<$ http://example.com/Ch\#/abc_aaa >ex:first

"abc"

$<$ http://example.com/Ch\#/abc_aaa>ex:second "aaa"

RDF output example graph

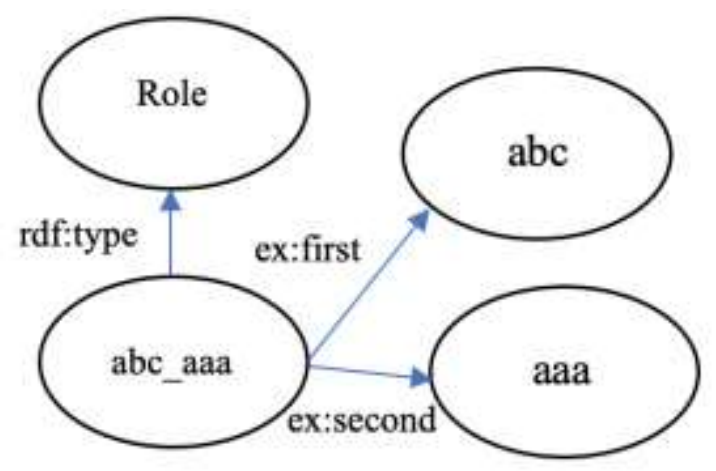

\subsection{Concept negation}

$\mathrm{RA}^{2}$ term

$(\neg \mathrm{C})_{\mathrm{RM}^{2}}^{\mathrm{E}}=\pi_{\mathrm{Name}}($ CIndividual $)-\mathrm{C}_{\mathrm{RM}^{2}}^{\mathrm{E}}$

\section{$\underline{\text { R2R ML Triple Map }}$}

$<\#$ TriplesMap19>

rr:logicalTable [ rr:sq1Query

“"” SELECT ci.Name

FROM CIndividual ci

EXCEPT
SELECT ci.Name

FROM CIndividual ci, Concept c, LinkCI lci, WHERE ci.CIPK $=1$ lci.CIFK. AND 1ci.CFK $=$ c.CPK AND c.Name $={ }^{\prime} \mathrm{C}$ ' '“"'];

r:subjectMap [

rr:template

«http://example.com/Ch\#/ $\{\mathrm{Name}\} » ;$ rr:class ex:CIndividual; ]

rr:predicateObjectMap [ rr:predicate ex:name rr:objectMap [ rr:column: Name;]

]

RDF output example

$<$ http://example.com/Ch\#/CIndividual/ccc > rdf:type ex:CIndividual.

$<$ http://example.com/Ch\#/CIndividual/ccc> ex:name "ccc".

RDF output example graph

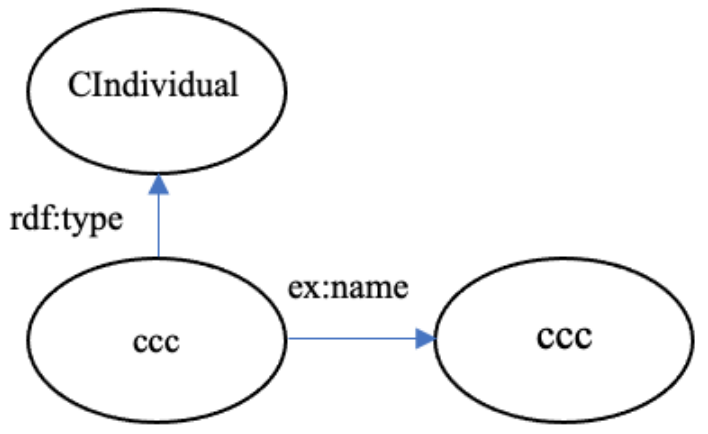

\subsection{Concept union}

\section{$\mathrm{RA}^{2}$ term}

$(\mathrm{C} \sqcup \mathrm{D})=\mathrm{C}_{\mathrm{RM}^{2}}^{\mathrm{E}} \cup \mathrm{D}_{\mathrm{RM}^{2}}^{\mathrm{E}}$

\section{$\underline{\text { R2R ML Triple Map }}$}

$<\#$ TriplesMap20>

rr:logicalTable [ rr:sqlQuery

“"”' SELECT ci.Name

FROM CIndividual ci, Concept c, LinkCI lci,

WHERE ci.CIPK $=$ lci.CIFK. AND lci.CFK $=$ c.CPK AND c.Name $=$ ' $\mathrm{C}$ '

UNION

SELECT ci.Name

FROM CIndividual ci, Concept c, LinkCI lci,

WHERE ci.CIPK $=$ 1ci.CIFK. AND 1ci.CFK $=$ c.CPK AND c.Name = ' $\mathrm{D}$ ' “'”'];

r:subjectMap [

rr:template 


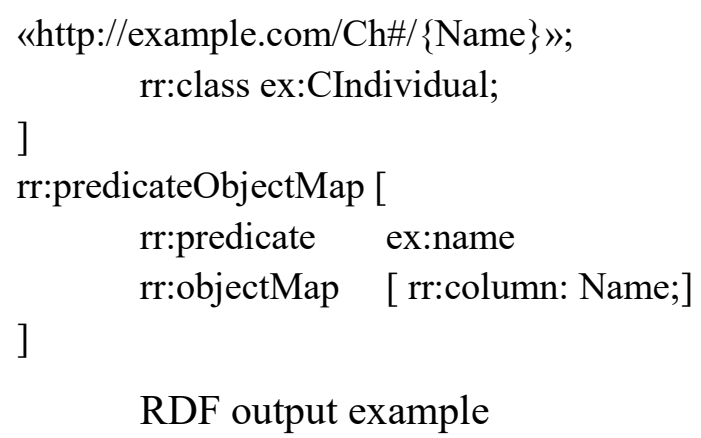

RDF output example

<http://example.com/Ch\#/CIndividual/bbb> rdf:type ex:CIndividual.

<http://example.com/Ch\#/CIndividual/bbb> ex:name "bbb".

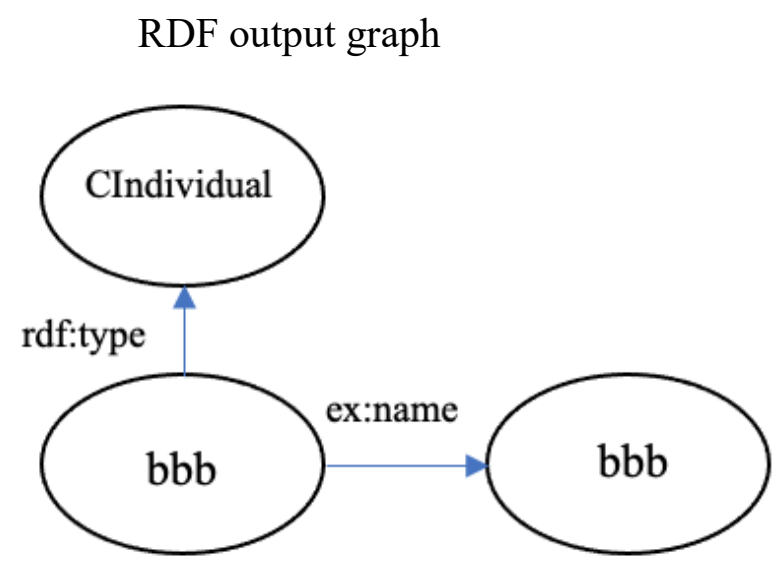

\subsection{Concept intersection}

$\mathrm{RA}^{2}$ term

$(\mathrm{C} \cap \mathrm{D})=\mathrm{C}_{\mathrm{RM}^{2}}^{\mathrm{E}} \cap \mathrm{D}_{\mathrm{RM}^{2}}^{\mathrm{E}}$

\section{R2R ML Triple Map}

$<$ \#TriplesMap21>

rr:logicalTable [ rr:sq1Query

“"”,

\section{SELECT ci.Name}

FROM CIndividual ci, Concept c, LinkCI lci, WHERE ci.CIPK $=1$ 1ci.CIFK. AND 1ci.CFK $=$ c. CPK AND c. Name $=$ ' $\mathrm{C}$ '

INTERSECT

SELECT ci.Name

FROM CIndividual ci, Concept c, LinkCI lci, WHERE ci.CIPK $=$ 1ci.CIFK. AND 1ci.CFK $=$ c. CPK AND c. Name $=$ ' $D$ '

“"'"];

r:subjectMap [

rr:template

«http://example.com/Ch\#/\{Name\}»;

rr:class ex:CIndividual;
rr:predicateObjectMap [

rr:predicate ex:name

rr:objectMap [ rr:column: Name;]

]

RDF output example

$<$ http://example.com/Ch\#/CIndividual/def> rdf:type ex:CIndividual.

$<$ http://example.com/Ch\#/CIndividual/def> ex:name "def".

RDF output example graph

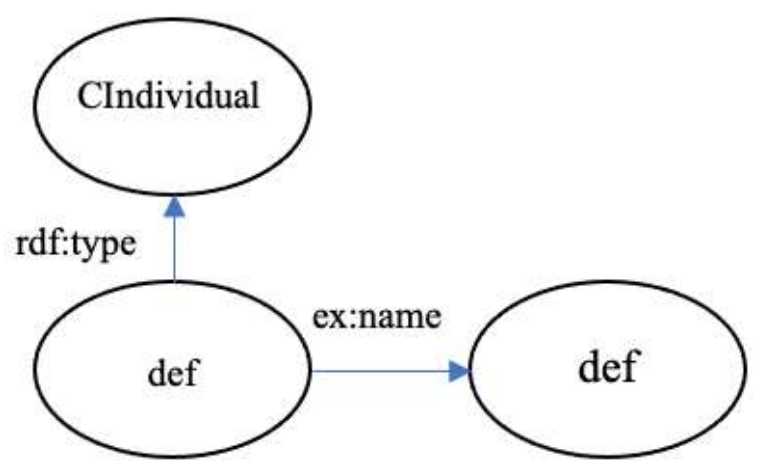

The following notation is introduced:

- a table RE(First, Second) was get after mapping $\mathrm{R}_{\mathrm{RM}^{2}}^{\mathrm{E}}(<\#$ TriplesMap18>);

- a table CE(Name) was get after mapping $\mathrm{C}_{\mathrm{RM}^{2}}^{\mathrm{E}}(<\#$ TriplesMap17>).

\subsection{Existential quantification}

$\mathrm{RA}^{2}$ term

$$
\begin{aligned}
(\exists \text { R. C })_{\mathrm{RM}^{2}}^{\mathrm{E}}= & \pi_{\text {First }}\left(\mathrm{R}_{\mathrm{RM}}^{\mathrm{E}}\right. \\
& \left.\bowtie_{\text {Second }=\text { Name }} \mathrm{C}_{\mathrm{RM}^{2}}^{\mathrm{E}}\right)
\end{aligned}
$$

\section{$\underline{\text { R2R ML Triple Map }}$}

<\#TriplesMap22>

rr:logicalTable [ rr:sqlQuery

“'”' SELECT RE.First

FROM RE, CE

WHERE RE.Second = CE.Name “"”'];

r:subjectMap [

rr:template

«http://example.com/Ch\#/\{First $\} » ;$ rr:class ex:CIndividual;

]

rr:predicateObjectMap [

rr:predicate ex:first 

]

rr:objectMap [ rr:column: First;]

RDF output example

<http://example.com/Ch\#/CIndividual/bbb> rdf:type ex:CIndividual.

<http://example.com/Ch\#/CIndividual/bbb> ex:name "bbb".

RDF output graph

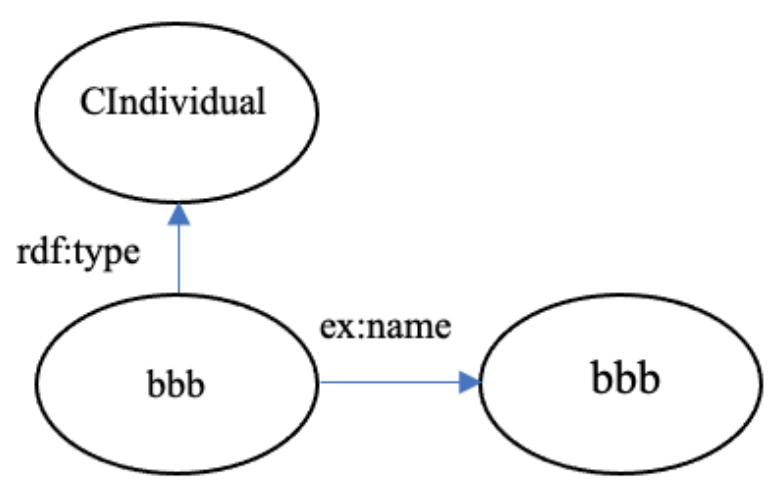

4.7. Value restriction

$\mathrm{RA}^{2}$ term

$(\forall \text { R. C })_{\mathrm{RM}^{2}}^{\mathrm{E}}=\pi_{\text {First }} \mathrm{R}_{\mathrm{RM}^{2}}^{\mathrm{E}}-\pi_{\text {First }}\left(\mathrm{R}_{\mathrm{RM}^{2}}^{\mathrm{E}}\right.$

$$
\begin{aligned}
& \cap\left(\pi_{\text {First }} R_{\mathrm{RM}^{2}}^{\mathrm{E}}\right. \\
& \times\left(\pi_{\text {Second }} \mathrm{R}_{\mathrm{RM}^{2}}^{\mathrm{E}}\right. \\
& \left.\left.-\pi_{\text {Name }} C_{\mathrm{RM}^{2}}^{\mathrm{E}}\right)\right)
\end{aligned}
$$

\section{R2R ML Triple Map}

<\#TriplesMap23>

rr:logicalTable [ rr:sqlQuery

“"'SELECT RE.First

FROM RE

EXCEPT

SELECT First

FROM (SELECT

FROM RE

INTERSECT

(SELECT *

FROM (SELECT RE.First

FROM RE),

(SELECT RE.Second

FROM RE

EXCEPT

SELECT CE.Name

r:subjectMap [ FROM CE))) '“"'];

rr:template «http://example.com/Ch\#/ FFirst $\} » ;$ rr:class ex:CIndividual;

]

rr:predicateObjectMap [

rr:predicate ex:first

rr:objectMap [ rr:column: First;

]

RDF output example

<http://example.com/Ch\#/CIndividual/aaa> rdf:type ex:CIndividual.

<http://example.com/Ch\#/CIndividual/aaa> ex:first "aaa".

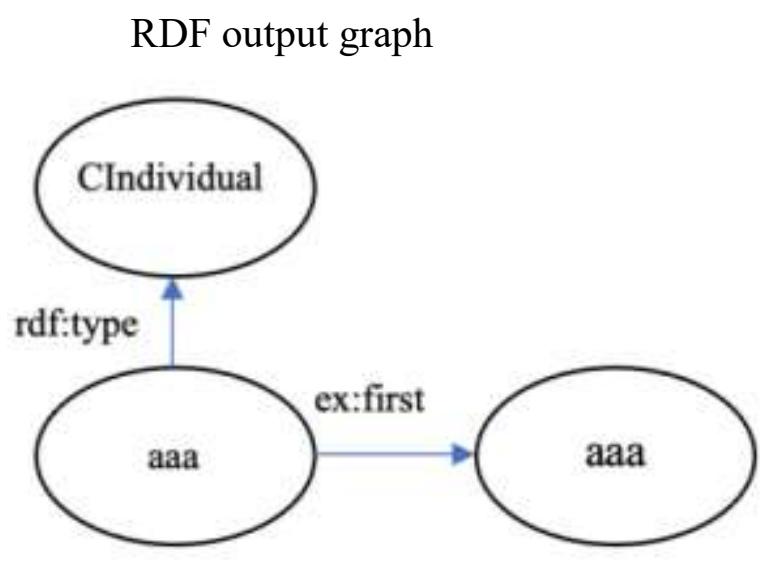

\section{ALC extensions}

The mappings to only several number restrictions are shown in the paper. Mappings for the rest of extensions uses the recursive SQL.

\subsection{Functional restrictions}

$$
\mathrm{RA}^{2} \text { term }
$$

$(\geq 2 \mathrm{R})_{\mathrm{RM}^{2}}^{\mathrm{E}}=\pi_{\text {First }}\left(\sigma_{\text {Second } 1 \neq \text { Second } 2}\right.$

( $\rho_{\text {Second/Second1 }}\left(\mathrm{R}_{\mathrm{RM}^{2}}^{\mathrm{E}}\right) \bowtie_{\text {First=First }}$

$\left.\left(\rho_{\text {Second/Second2 }}\left(\mathrm{R}_{\mathrm{RM}^{2}}^{\mathrm{E}}\right)\right)\right)$ )

\section{$\underline{\text { R2R ML Triple Map }}$}

<\#TriplesMap24>

rr:logicalTable [ rr:sqlQuery

"“"SELECT one.First

FROM RE one, RE two

WHERE one. First $=$ two.First

AND one.Second <> two.Second "'"'];

r:subjectMap [

rr:template «http://example.com/Ch\#/\{First\}»; 
rr:class ex:CIndividual;

]

rr:predicateObjectMap [

rr:predicate ex:first

rr:objectMap [rr:column: First;

]

RDF output example

<http://example.com/Ch\#/CIndividual/abc> rdf:type ex:CIndividual.

<http://example.com/Ch\#/CIndividual/abc> ex:name "abc".

RDF output graph

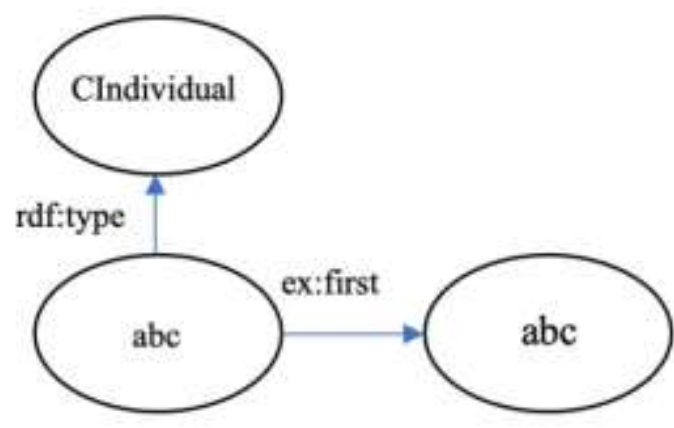

$\mathrm{RA}^{2}$ term

$(\leq 1 R)_{R M^{2}}^{E}=\pi_{\text {Name }}($ CIndividual $)$

$-(\geq 2 R)_{R M^{2}}^{E}$

\section{$\underline{\text { R2R ML Triple Map }}$}

<\#TriplesMap25>

rr:logicalTable [ rr:sqlQuery

“"'SELECT ci.Name

FROM CIndividual ci

\section{EXCEPT}

SELECT one.First

FROM RE one, RE two

WHERE one.First $=$ two.First

AND one.Second $<>$ two.Second "'"'];

r:subjectMap [

rr:template «http://example.com/Ch\#/ Name $\} »$;

rr:class ex:CIndividual;

]

rr:predicateObjectMap [

rr:predicate ex:name

rr:objectMap [ rr:column: Name;]
RDF output example

<http://example.com/Ch\#/CIndividual/ccc> rdf:type ex:CIndividual.

<http://example.com/Ch\#/CIndividual/ccc> ex:name "ccc".

RDF output graph

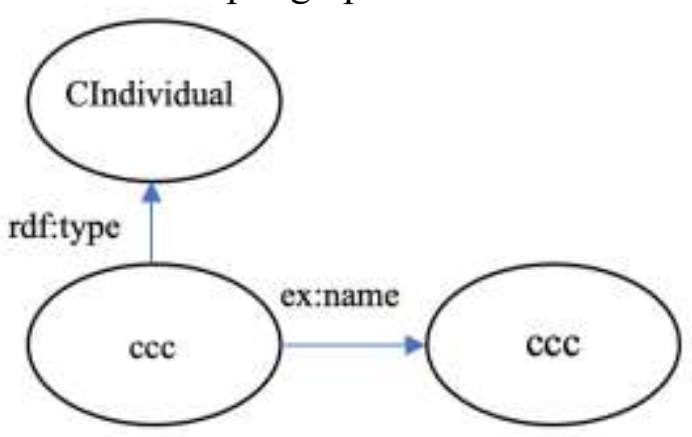

5.2. Several quality restrictions

$\mathrm{RA}^{2}$ term

$(\geq 2 \mathrm{R} . \mathrm{C})_{\mathrm{RM}^{2}}^{\mathrm{E}}=$

$\pi_{\text {First }}\left(\sigma_{\text {Second }_{1} \neq \text { Second }_{2}}\left(\rho_{\text {Second }_{\text {Second }}}\right.\right.$

$\left(\mathrm{R}_{\mathrm{RM}^{2}}^{\mathrm{E}}\right) \bowtie_{\text {Second }=\text { Name }}\left(\rho_{\text {Second } / \text { Second }_{2}}\right.$

$\left.\left.\left(\mathrm{R}_{\mathrm{RM}^{2}}^{\mathrm{E}} \bowtie_{\text {Second=Name }} \mathrm{C}_{\mathrm{RM}^{2}}^{2}\right)\right)\right)$

R2R ML Triple Map

<\#TriplesMap26>

rr:logicalTable [ rr:sqlQuery

“"'SELECT one.First

FROM

(SELECT First, Second

FROM RE, CE

WHERE RE.Second = CE.Name) one, (SELECT First, Second

FROM RE, CE

WHERE RE.Second = CE.Name) two

WHERE one,First $=$ two.First

AND one.Second <> two.Second“"'"];

r:subjectMap [

rr:template «http://example.com/Ch\#/\{First $\} » ;$ rr:class ex:CIndividual;

]

rr:predicateObjectMap [

rr:predicate ex:first

rr:objectMap [ rr:column: First;] 
RDF output example

〈http://example.com/Ch\#/CIndividual/aaa>

rdf:type ex:CIndividual.

<http://example.com/Ch\#/CIndividual/aaa> ex:first "aaa".

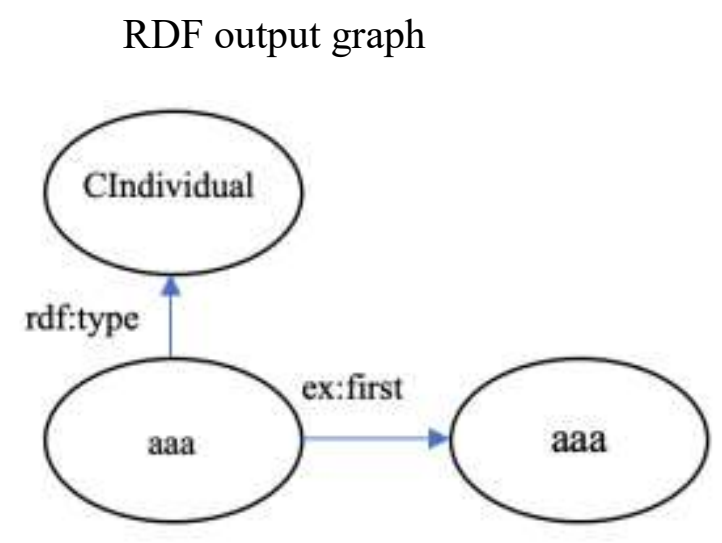

$\mathrm{RA}^{2}$ term

$$
\begin{gathered}
(\leq 1 R)_{R M^{2}}^{E}=\pi_{\text {Name }}(\text { CIndividual }) \\
-(\geq 2 R . C)_{R M^{2}}^{E}
\end{gathered}
$$

\section{$\underline{\text { R2R ML Triple Map }}$}

<\#TriplesMap27>

rr:logicalTable [ rr:sqlQuery

“"'SELECT ci.Name

FROM CIndividual ci

EXCEPT

\section{SELECT one.First}

FROM

(SELECT First, Second

FROM RE, CE

WHERE RE.Second = CE.Name) one,

(SELECT First, Second

FROM RE, CE

WHERE RE.Second = CE.Name) two

WHERE one,First $=$ two. First

AND one.Second $<>$ two.Second "'"'];

r:subjectMap [

rr:template «http://example.com/Ch\#/\{Name $\} » ;$

rr:class ex:CIndividual;

]

rr:predicateObjectMap [

rr:predicate ex:name

rr:objectMap [ rr:column: Name;]
RDF output example

<http://example.com/Ch\#/CIndividual/def> rdf:type ex:CIndividual.

<http://example.com/Ch\#/CIndividual/def> ex:name "def".

RDF output graph

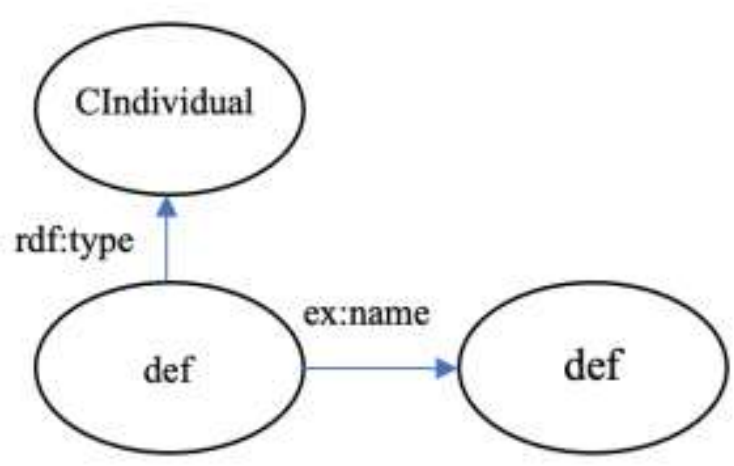

\section{Role restrictions}

Despite the number of role restrictions only role inverse mapping into RDF is considered in the paper. As known [19] the OWL 2 is based on the DL SROIQ. This logic includes only role inverse through all the role restrictions amount. So, the mapping for other role restrictions is out of scope of this research.

$$
\begin{gathered}
\text { 6.1. Role inverse } \\
\mathrm{RA}^{2} \text { term } \\
\left(\mathrm{R}^{-}\right)_{\mathrm{RM}^{2}}^{\mathrm{E}}=\left(\rho_{\mathrm{R}(\text { Second,First })}\left(\mathrm{R}_{\mathrm{RM}^{2}}^{\mathrm{E}}\right)\right)
\end{gathered}
$$

$\underline{\text { R2R ML Triple Map }}$

<\#TriplesMap28>

rr:logicalTable [ rr:sqlQuery

“"'"SELECT second.Name AS First, first.Name AS Second

FROM Role r, RIndividual ri, LinkRRI lrri, Predecessor p, Successor s

CIndividual first, CIndividual.second

WHERE r.RPK $=$ 1rri.RFK AND lrri.RIFK $=$ ri.RIPK AND ri.RIPK $=$ p.RIFK AND p.CIFK $=$ first.CIPK AND ri.RIPK $=$ s.RIFK AND s.CIFK = second. CIPK AND r.Name='R" "'"']; r:subjectMap [ 
rr:template «http://example.com/Ch\#/\{Name\}»; rr:class ex:Role;

]

rr:predicateObjectMap [

$$
\begin{array}{ll}
\text { rr:predicate } & \text { ex:name } \\
\text { rr:objectMap } & \text { [ rr:column: Name; }
\end{array}
$$

]

\section{RDF output example}

$<$ http://example.com/Ch\#/aaa_abc >rdf:type ex:Role;

$<$ http://example.com/Ch\#/aaa_abc>ex:first

"aaa"

$<$ http://example.com/Ch\#/aaa_abc>ex:second " $a b c "$

RDF output example graph

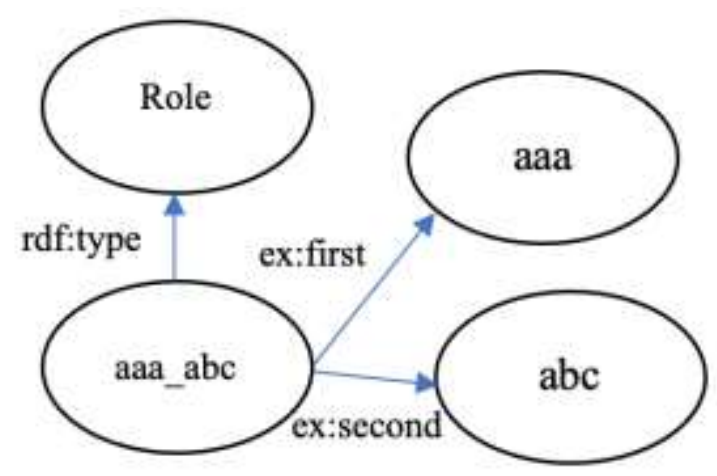

\section{Conclusions}

The article outlines a method how to check mappings between the descriptive logic and the binary relational data model using mappings into RDF. The task is set. The description of the theoretical aspects is presented. The publication provides mappings for the binary relational data model into an RDF triples using the R2R ML language. The paper also outlines the rules for converting the DLto- $\mathrm{RM}^{2}$ mapping formulas into RDF.

The issue of converting a number of role constructors into RDF remains open.

\section{References}

1. Chystiakova, I. Ontology-oriented data integration on the Semantic Web. Problems in Programming. 2014. N 2-3. P. 188-196.
2. Reznichenko, V. and Chystiakova, I. Mapping of the Description Logics ALC into the Binary Relational Data Structure. Problems in Programming. 2015. N 4. P. 13-30.

3. Reznichenko, V. and Chystiakova, I. Integration of the family of extended description logics with relational data model. Problems in Programming. (2016). N 2-3. P. 38-47.

4. Chystiakova, I. Integration of the description logics with extensions into relational data model. Problems in Programming. 2016. N 4. P. 58-65.

5. Chystiakova, I. Integration of the Descriptive Logic Axiomatics with the Relational Data Model. Problems in programming. 2017. N 1. P. 51-58.

6. Reznichenko, V. and Chystiakova, I. Binary Relational Data Model. Problems in Programming. 2017. N 2 (4). P. 96-105.

7. Chystiakova I.S. (2018). Mapping of the Relational Algebra into Descriptive Logic. Problems in Programming. 2017. N 2-3. P. $214-225$.

8. Andon P. and Reznichenko V. and Chystiakova I. Mapping of Description Logic to the Relational Data Model. Cybernetics and Systems Analysis. 2017. N 53 (6). P. 963-978.

9. Chystiakova I.S. Implementation of mappings between the description logic and the binary relational data model on the RDF level. Problems in programming. 2020. N 4. P. $41-54$.

10. Heath I.J. Unacceptable file operations in a relational data base. SIGFIDET '71. 1971. P. 19-33.

11. OWL 2 Web Ontology Language. Mapping to RDF Graphs (Second Edition). [Online] December 2012. Available from: https://www.w3.org/TR/owl2-mapping-tordf/\#Translation_of_Axioms_without_Annota tions. [Accessed: 20 February 2021].

12. R2RML: RDB to RDF Mapping Language. [Online] September 2012. Available from: https://www.w3.org/TR/r2rml/. (last access 20 February 2021).

13. Caroll J.J. Matching RDF Graphs I. Horrocks and J. Hendler (Eds.): ISWC. 2002. LNCS 2342. P. 5-15.

14. Berners-Lee, T. Relational Databases on the Semantic Web [Online] September 1998. Available from: https://www.w3.org/ DesignIssues/RDB-RDF.html. (last access 20 February 2021).

15. W3C Workshop on RDF Access to Relational Databases URL: 
https://www.w3.org/2007/03/RdfRDB/ (last access 20 February 2021)

16. RDB2RDF Tutorial (R2RML and Direct Mapping) at ISWC 2013 URL: https://www.slideshare.net/juansequeda/rdb2rdf-tutorial-iswc2013 (last access 20 February 2021)

17. Codd E.F. Extending the database relational model to capture more meaning. ACM Transactions on Database Systems (TODS). 1979. Vol. 4. Issue 4. P. 397-434.

18. Barker R. Case* method: entity relationship modelling. Addison-Wesley. 1990. P. 240.

19. The description logic handbook: theory, implementation, and applications. Baader F., Calvanese D., McGuinness D., Nardi D., Patel-Schneider P. (Eds.) Cambridge University Press. 2003. 555 p.

\section{Література}

1. Чистякова I.С. Онтолого-ориентированная интеграция данных в семантическом вебе. Проблеми програмування. 2014. № 2-3. C. $188-196$.

2. Резниченко В.А., Чистякова И.С. Отображение дескриптивной логики ALC в бинарную реляционную структуру данных. Проблеми програмування. 2015. № 4 . С. 13-30.

3. Резниченко В.А., Чистякова И.С. Интеграция семейства расширенных дескриптивных логик с реляционной моделью данных. Проблеми програмування. 2016. № 2-3. C. 38-47.

4. Чистякова И.С. Интеграция логик с операциями над ролями с реляционной моделью данных. Проблеми програмування. 2016. № 4. C. 58-65.

5. Чистякова И.С. Интеграция аксиоматики дескриптивных логик с реляционной моделью данных. Проблеми програмування. 2017. № 1. C. 51 - 58.

6. Резниченко В.А., Чистякова И.С. Бинарная реляционная модель данных. Проблеми програмування. 2017. № 2. С. 96 - 105.

7. Чистякова И.С. Отображение реляционной алгебры в дескриптивную логику. Проблеми програмування. 2018. № 2-3. C. $214-225$.

8. Andon P. and Reznichenko V. and Chystiakova I. Mapping of Description Logic to the Relational Data Model. Cybernetics and
Systems Analysis. 2017. N 53 (6). P. 963-978.

9. Chystiakova I.S. Implementation of mappings between the description logic and the binary relational data model on the RDF level. Problems in programming. 2020. N 4. P. $41-54$.

10. Heath I.J. Unacceptable file operations in a relational data base. SIGFIDET '71. 1971. P. 19-33.

11. OWL 2 Web Ontology Language. Mapping to RDF Graphs (Second Edition). [Online] December 2012. Available from: https://www.w3.org/TR/owl2-mapping-tordf/\#Translation_of_Axioms_without_Annota tions. [Accessed: 20 February 2021].

12. R2RML: RDB to RDF Mapping Language. [Online] September 2012. Available from: https://www.w3.org/TR/r2rml/. (last access 20 February 2021).

13. Caroll J.J. Matching RDF Graphs I. Horrocks and J. Hendler (Eds.): ISWC. 2002. LNCS 2342. P. 5-15.

14. Berners-Lee, T. Relational Databases on the Semantic Web [Online] September 1998. Available from: https://www.w3.org/ DesignIssues/RDB-RDF.html. (last access 20 February 2021).

15. W3C Workshop on RDF Access to Relational Databases URL: https://www.w3.org/2007/03/RdfRDB/ (last access 20 February 2021)

16. RDB2RDF Tutorial (R2RML and Direct Mapping) at ISWC 2013 URL: https://www.slideshare.net/juansequeda/rdb2rdf-tutorial-iswc2013 (last access 20 February 2021)

17. Codd E.F. Extending the database relational model to capture more meaning. ACM Transactions on Database Systems (TODS). 1979. Vol. 4. Issue 4. P. 397-434.

18. Barker R. Case* method: entity relationship modelling. Addison-Wesley. 1990. P. 240.

19. The description logic handbook: theory, implementation, and applications. Baader F., Calvanese D., McGuinness D., Nardi D., Patel-Schneider P. (Eds.) Cambridge University Press. 2003. 555 p. 


\section{About the author:}

Inna Chystiakova, junior researcher at the Institute of software systems of NASU.

The number of publications in Ukrainian journals is 11 .

The number of publications in foreign journals is 1 .

Hirsh index is 5.

https://orcid.org/0000-0001-7946-3611.

\section{Affiliation:}

Institute of software systems of NASU 03187, Kyiv,

pr. Glushkova, 40, build 5.

Tel.: +38(066)8477784.

E-mail: inna_islyamova@ukr.net. 